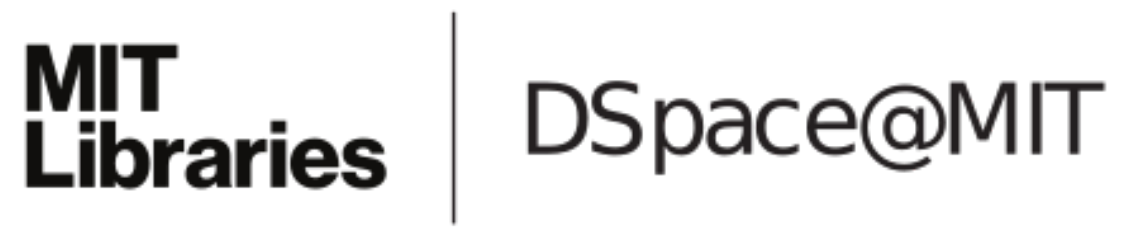

\author{
MIT Open Access Articles
}

\section{Warm Jupiters in TESS Full-frame Images: A Catalog and Observed Eccentricity Distribution for Year 1}

The MIT Faculty has made this article openly available. Please share how this access benefits you. Your story matters.

As Published: 10.3847/1538-4365/abf73c

Publisher: American Astronomical Society

Persistent URL: https://hdl.handle.net/1721.1/135635

Version: Final published version: final published article, as it appeared in a journal, conference proceedings, or other formally published context

Terms of Use: Article is made available in accordance with the publisher's policy and may be subject to US copyright law. Please refer to the publisher's site for terms of use. 


\section{Warm Jupiters in TESS Full-frame Images: A Catalog and Observed Eccentricity Distribution for Year 1}

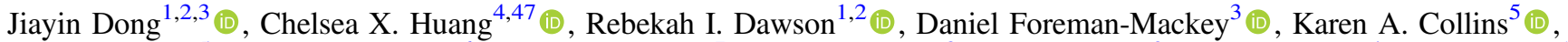

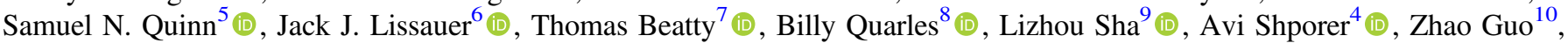
Stephen R. Kane ${ }^{11}$ (1), Lyu Abe ${ }^{12}$, Khalid Barkaoui ${ }^{13,14}$ (1) , Zouhair Benkhaldoun $^{14}$ (1), Rafael Brahm ${ }^{15,16}$ (1) , François Bouchy ${ }^{17}$ (1),

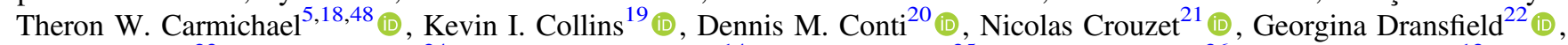

Phil Evans ${ }^{23}$ (1), Tianjun Gan ${ }^{24}$ (i), Mourad Ghachoui ${ }^{14}$, Michaël Gillon ${ }^{25}$ (i), Nolan Grieves ${ }^{26}$ (i), Tristan Guillot ${ }^{12}$ (i),

Coel Hellier ${ }^{27}$ (10), Emmanuël Jehin ${ }^{28}$ (D), Eric L. N. Jensen ${ }^{29}$ (D), Andres Jordán ${ }^{15,16}$ (1) , Jacob Kamler ${ }^{30}$, John F. Kielkopf ${ }^{31}$ (10), Djamel Mékarnia ${ }^{12}$ (10), Louise D. Nielsen ${ }^{17}$ (1) Francisco J. Pozuelos ${ }^{25,28}$ (1) , Don J. Radford ${ }^{32}$, François-Xavier Schmider ${ }^{12}$ (i), Richard P. Schwarz ${ }^{33}$ (1), Chris Stockdale ${ }^{34}$ (1), Thiam-Guan $\operatorname{Tan}^{35}$ (1), Mathilde Timmermans ${ }^{25}$, Amaury H. M. J. Triaud ${ }^{22}$ (1), Gavin Wang ${ }^{36,37}$ (1) , George Ricker ${ }^{4}$ (1) , Roland Vanderspek ${ }^{4}$ (1), David W. Latham ${ }^{18}$ (10), Sara Seager ${ }^{4,38,39}$ (1) , Joshua N. Winn ${ }^{40}$ (1),

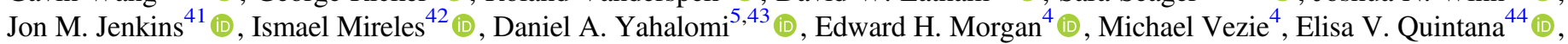
Mark E. Rose ${ }^{41}$ (D) Jeffrey C. Smith ${ }^{41,45}$ (1), and Bernie Shiao ${ }^{46}$

\footnotetext{
${ }^{1}$ Department of Astronomy \& Astrophysics, The Pennsylvania State University, University Park, PA 16802, USA; jdong@psu.edu

${ }^{2}$ Center for Exoplanets \& Habitable Worlds, 525 Davey Laboratory, The Pennsylvania State University, University Park, PA 16802, USA

${ }^{3}$ Center for Computational Astrophysics, Flatiron Institute, 162 Fifth Avenue, New York, NY 10010, USA

${ }^{4}$ Department of Physics and Kavli Institute for Astrophysics and Space Research, Massachusetts Institute of Technology, Cambridge, MA 02139, USA

${ }^{5}$ Center for Astrophysics | Harvard \& Smithsonian, 60 Garden Street, Cambridge, MA 02138, USA

${ }^{6}$ Space Science \& Astrobiology Division, MS 245-3, NASA Ames Research Center, Moffett Field, CA 94035, USA

${ }^{7}$ Department of Astronomy and Steward Observatory, University of Arizona, Tucson, AZ 85721, USA

${ }^{8}$ Center for Relativistic Astrophysics, School of Physics, Georgia Institute of Technology, Atlanta, GA 30332 USA

${ }_{9}$ Department of Astronomy, University of Wisconsin-Madison, Madison, WI 53706, USA

${ }^{10}$ Department of Applied Mathematics and Theoretical Physics (DAMTP),University of Cambridge, UK

${ }^{11}$ Department of Earth and Planetary Sciences, University of California, Riverside, CA 92521, USA

${ }^{12}$ Université Côte d'Azur, Observatoire de la Côte d'Azur, CNRS, Laboratoire Lagrange, Bd de l'Observatoire, CS 34229, F-06304 Nice cedex 4, France

${ }^{13}$ Astrobiology Research Unit, Université de Liége, 19C Allée du 6 Août, B-4000 Liège, Belgium

${ }^{14}$ Oukaimeden Observatory, High Energy Physics and Astrophysics Laboratory, Cadi Ayyad University, Marrakech, Morocco

${ }^{15}$ Facultad de Ingeniería y Ciencias, Universidad Adolfo Ibáñez, Av. Diagonal las Torres 2640, Peñalolén, Santiago, Chile

${ }^{16}$ Millennium Institute for Astrophysics, Chile

${ }^{17}$ Observatoire de l'Université de Genève, 51 chemin des Maillettes, 1290 Versoix, Switzerland

18 Department of Astronomy, Harvard University, Cambridge, MA 02138, USA

${ }^{19}$ George Mason University, 4400 University Drive, Fairfax, VA, 22030 USA

${ }^{20}$ American Association of Variable Star Observers, 49 Bay State Road, Cambridge, MA 02138, USA

${ }^{21}$ European Space Agency (ESA), European Space Research and Technology Centre (ESTEC), Keplerlaan 1, 2201 AZ Noordwijk, The Netherlands

${ }^{2}$ School of Physics \& Astronomy, University of Birmingham, Edgbaston, Birmingham B15 2TT, UK

${ }^{23}$ El Sauce Observatory, Coquimbo Province, Chile

${ }^{24}$ Department of Astronomy and Tsinghua Centre for Astrophysics, Tsinghua University, Beijing 100084, People's Republic of China

${ }_{25}$ Astrobiology Research Unit, Université de Liège, 19C Allèe du 6 Août, B-4000 Liège, Belgium

${ }^{26}$ Observatoire de Genève, Université de Genève, Chemin Pegasi 51b, 1290 Sauverny, Switzerland ${ }^{27}$ Astrophysics Group, Keele University, Staffordshire ST5 5BG, UK

${ }^{28}$ Space Sciences, Technologies and Astrophysics Research (STAR) Institute, Université de Liège, 19C Allèe du 6 Août, B-4000 Liège, Belgium

${ }^{29}$ Department of Physics \& Astronomy, Swarthmore College, Swarthmore PA 19081, USA

30 John F. Kennedy High School, 3000 Bellmore Avenue, Bellmore, NY 11710, USA

${ }^{31}$ Department of Physics and Astronomy, University of Louisville, Louisville, KY 40292, USA

${ }^{32}$ Brierfield Observatory, New South Wales, Australia

${ }^{33}$ Patashnick Voorheesville Observatory, Voorheesville, NY 12186, USA

${ }^{34}$ Hazelwood Observatory, Australia

${ }^{35}$ Perth Exoplanet Survey Telescope, Perth, Western Australia, Australia

${ }^{36}$ Stanford Online High School, 415 Broadway Academy Hall, Floor 2, 8853, Redwood City, CA 94063, USA

${ }^{37}$ Tsinghua International School, Beijing 100084, People's Republic of China

${ }^{38}$ Department of Earth, Atmospheric and Planetary Sciences, Massachusetts Institute of Technology, Cambridge, MA 02139, USA

${ }^{39}$ Department of Aeronautics and Astronautics, MIT, 77 Massachusetts Avenue, Cambridge, MA 02139, USA

${ }^{40}$ Department of Astrophysical Sciences, Princeton University, NJ 08544, USA

${ }^{41}$ NASA Ames Research Center, Moffett Field, CA 94035, USA

42 Department of Physics and Astronomy, University of New Mexico, 210 Yale Blvd NE, Albuquerque, NM 87106, USA

${ }^{43}$ Department of Astronomy, Columbia University, 550 West 120th Street, New York, NY 10027, USA

${ }^{44}$ NASA Goddard Space Flight Center, 8800 Greenbelt Road, Greenbelt, MD 20771, USA

${ }^{45}$ SETI Institute, 189 Bernardo Ave, Mountain View, CA 94043, USA

${ }^{46}$ Mikulski Archive for Space Telescopes, 3700 San Martin Drive, Baltimore, MD 21218, USA

Received 2021 January 25; revised 2021 April 2; accepted 2021 April 10; published 2021 June 28
}

\footnotetext{
47 Juan Carlos Torres Fellow.

${ }^{48}$ National Science Foundation Graduate Research Fellow.
} 


\begin{abstract}
Warm Jupiters — defined here as planets larger than 6 Earth radii with orbital periods of 8-200 days-are a key missing piece in our understanding of how planetary systems form and evolve. It is currently debated whether Warm Jupiters form in situ, undergo disk or high-eccentricity tidal migration, or have a mixture of origin channels. These different classes of origin channels lead to different expectations for Warm Jupiters' properties, which are currently difficult to evaluate due to the small sample size. We take advantage of the Transiting Exoplanet Survey Satellite (TESS) survey and systematically search for Warm Jupiter candidates around main-sequence host stars brighter than the TESS-band magnitude of 12 in the full-frame images in Year 1 of the TESS Prime Mission data. We introduce a catalog of 55 Warm Jupiter candidates, including 19 candidates that were not originally released as TESS objects of interest by the TESS team. We fit their TESS light curves, characterize their eccentricities and transit-timing variations, and prioritize a list for ground-based follow-up and TESS Extended Mission observations. Using hierarchical Bayesian modeling, we find the preliminary eccentricity distributions of our Warm-Jupiter-candidate catalog using a beta distribution, a Rayleigh distribution, and a two-component Gaussian distribution as the functional forms of the eccentricity distribution. Additional follow-up observations will be required to clean the sample of false positives for a full statistical study, derive the orbital solutions to break the eccentricity degeneracy, and provide mass measurements.
\end{abstract}

Unified Astronomy Thesaurus concepts: Exoplanet catalogs (488)

Supporting material: figure set

\section{Introduction}

We do not yet understand the formation of Warm Jupiters. Although intrinsically uncommon relative to other planetary demographics (e.g., Jones et al. 2003; Udry et al. 2003; Wittenmyer et al. 2010; Santerne et al. 2016), they are a result of physical processes that likely sculpt many planetary systems, and are excellent test cases for theories inspired by the more frequently detected hot Jupiters (i.e., gas giants with orbital periods less than 8 days) and Warm mini-Neptunes/superEarths (i.e., planets between the sizes of Earth and Neptune with orbital periods of 8-200 days). It is currently debated whether Warm Jupiters form in situ (e.g., Lee et al. 2014; Batygin et al. 2016; Boley et al. 2016; Lee \& Chiang 2016) or undergo disk migration (e.g., Goldreich \& Tremaine 1980; Lin \& Papaloizou 1986; Lin et al. 1996; Ida \& Lin 2008; Baruteau et al. 2014) or high-eccentricity tidal migration (e.g., Rasio \& Ford 1996; Wu \& Lithwick 2011; Petrovich 2015; see Section 4.3 of Dawson \& Johnson 2018 for a comprehensive review). Three proposed origin channels lead to different expectations for Warm Jupiters' masses (e.g., Ida \& Lin 2008; Lee et al. 2014; Lee \& Chiang 2016), eccentricities (e.g., Duffell \& Chiang 2015; Petrovich \& Tremaine 2016; Frelikh et al. 2019; Anderson et al. 2020), host-star obliquities (e.g., Naoz et al. 2012; Li \& Winn 2016; Petrovich \& Tremaine 2016) and metallicities (e.g., Dawson \& Murray-Clay 2013; Tsang et al. 2014), and the presence and properties of other planets in the system (e.g., Dong et al. 2014; Huang et al. 2016). Recently, the diversity in these properties has inspired the hypothesis that multiple origin channels contribute substantially to the Warm Jupiter population (e.g., Dawson \& Johnson 2018).

The Transiting Exoplanet Survey Satellite (TESS; Ricker et al. 2015) provides an excellent opportunity to examine these hypotheses for Warm Jupiters' origins. Using TESS data, a handful of Warm Jupiter systems (e.g., TOI-172, TOI-216, TOI481, TOI-677, and TOI-1130; Dawson et al. 2019; Kipping et al. 2019; Rodriguez et al. 2019; Brahm et al. 2020; Jordán et al. 2020; Huang et al. 2020a) have been discovered and characterized, allowing us to examine whether multiple properties of the system tell a consistent story about the system's origin (i.e., whether each property is consistent with the same origin theory). More generally, TESS is discovering a large sample of Warm
Jupiters in the full-frame images (FFIs) that allows for a statistical study of the Warm Jupiter population. Origin theories make different predictions for the eccentricity distribution and occurrence rates of Warm Jupiters (e.g., Petrovich \& Tremaine 2016), which are currently difficult to evaluate, due to their small sample size.

In this work, we describe a systematic search for Warm Jupiters, defined here as planets larger than 6 Earth radii with orbital periods of 8-200 days, around stars brighter than 12th TESS-band magnitude (Tmag) in the FFIs in the first year of TESS data. We note that the Warm Jupiters here can be more accurately termed warm, large planet candidates since we do not have their mass measurements. Planets larger than 6 Earth radii are likely to be gas giants, although we cannot rule out the possibility of super-puffs (i.e., large planets with low densities), which likely have different formation process. We construct a southern ecliptic hemisphere catalog of Warm Jupiter candidates in Year 1 of the TESS FFIs; prioritize a list of Warm Jupiter candidates showing evidence of strong transit-timing variations (TTVs) and high eccentricities, for ground-based follow-up and the TESS Extended Mission; and derive the eccentricity distribution of our Warm Jupiter candidate catalog using hierarchical Bayesian modeling (HBM), to compare to expectations of different origin theories.

In Section 2, we describe our pipeline for discovering Warm Jupiter candidates in TESS FFIs. In Section 3, we present our fitting model for TESS light curves and post fitting analysis. In Section 4, we catalog the resulting Warm Jupiter candidates discovered in Year 1 of the TESS FFIs, and highlight candidates showing possible TTV signals and evidence of high eccentricities. In Section 5, we infer the eccentricity distribution of the catalog using HBM. We put our work in the context of TESS Extended Mission and ground-based followup observations and discuss the implications of our results for origins of Warm Jupiters in Section 6. We summarize our findings in Section 7.

\section{Transit Search}

During the first year of the TESS Prime Mission (2018 July 25-2019 July 18), TESS surveyed almost the entire southern ecliptic hemisphere (Ricker et al. 2015). The Year 1 Prime 
Mission was divided into 13 TESS sectors (Sector 1-13), with each sector monitoring a fraction of sky for $\sim 27$ days. The TESS data release includes 30 minute cadence FFIs and 2 minute cadence for $\sim 200,000$ preselected target stars. Given the long orbital periods and low occurrence rates of Warm Jupiters, we should expect only a dozen or so Warm Jupiter candidates on the preselected list. Here, we systematically search for Warm Jupiter candidates in TESS FFIs, which include over a million stars brighter than Tmag of 12. Warm Jupiters' large transit depths $\left(\sim 10^{4} \mathrm{ppm}\right)$ make them readily detected in FFIs.

\subsection{Identifying Threshold-crossing Events}

We identify threshold-crossing events (TCEs) in TESS Sector 1-13 that would correspond to transits of objects with radii 6-20 $R_{\oplus}$, orbital periods 8-200 days, and transit signal-to-noise ratios (S/Ns) greater than 9 (see the definition of S/Ns in Hartman \& Bakos 2016). We focus on TCEs with host stars brighter than the Tmag of 12, to produce a catalog feasible for ground-based follow-up. The Tmag and parameter cutoff here are based on TESS Input Catalog v7 (TICv7; Stassun et al. 2018). The TICv8 catalog (Stassun et al. 2019) was not yet published when we made the target selection. All the TCEs are required to receive a triage score $>0.09$ (Yu et al. 2019), which is the threshold from the deep learning algorithm to distinguish eclipse-like signals (e.g., planet candidates and eclipsing binaries) from stellar variability and instrumental noise. After applying these selection criteria, we identify $\sim 2000$ TCEs.

\subsection{Vetting TCEs}

We further vet the $\sim 2000$ TCEs to remove false positives (e.g., obvious eclipsing binaries, stellar variability, and instrumental artifacts) that were not identified by triage, as well as single-transit events. ${ }^{49}$ The procedures are similar to standard vetting procedures applied to TESS objects of interest (TOIs; Guerrero et al. 2021). This process truncates our sample to $\sim 500$ Warm Jupiter candidates. For the remaining $\sim 500$ Warm Jupiter candidates, we examine more closely each candidate to identify those with signatures of false positives. We remove the target from our sample if it shows:

1. detectable motion of the center of the light during fading events;

2. $>3 \sigma$ secondary eclipse detection at any phase;

3. $>3 \sigma$ transit depth difference detection between a large aperture (3.5 pixel) and a nominal aperture (2.5 pixel);

4. transit signal synchronized with stellar variability;

5. matching with any known eclipsing binary catalogs (Collins et al. 2018);

6. impact parameter $b>0.9$.

For giant planets with a typical planet-star radius ratio of $\sim 0.1$, an impact parameter greater than 0.9 may lead to difficulty in constraining the planet-star radius ratio and impact parameter from their light curves. To minimize the contamination of eclipsing binaries in our sample, we remove these targets. The impact-parameter cutoff has a trade-off between false positives and real planets with high impact parameters. At the time of this writing, we notice the confirmation of a high-impact-parameter

\footnotetext{
49 A list of single-transit events is available from J.D. upon request, although we note that our detection pipeline (i.e., a box least-squares search) is not optimized for single-transit events.
}

Warm Jupiter, TIC 237913194 (Schlecker et al. 2020), which is not identified in our catalog due to the impact-parameter cutoff. One may also concern that the impact-parameter cutoff will result in removing candidates on highly elliptical orbits, because of their short transit durations. Since giant planets usually have large transit depths and well-resolved transit shapes, we do not expect the cutoff will strongly affect planets with high eccentricities. Finally, we remove about 15 targets that have been labeled as false positives by the TESS Follow-up Observing Program at the time of vetting. See Section 2.3 for more details.

Our study focuses on host stars that are on or near the main sequence and have TCEs around substantially evolved stars removed (i.e., $R_{\star}>3 R_{\odot}$ or $\log g<3.5$ ). We derive posteriors for stellar parameters (including $\rho_{\star}$ and $R_{\star}$ ) from isochrone fitting with the Dartmouth (Dotter et al. 2008) stellar evolution models. We use the approach described by Dawson et al. (2015) to fit the stellar effective temperature, metallicity (when available), Gaia DR2 parallax, and Gaia apparent $g$ magnitude (Gaia Collaboration et al. 2016, 2018). We apply the systematic correction to Gaia parallaxes from Stassun \& Torres (2018). The stellar temperature, metallicity, and uncertainties are taken from the TICv8 catalog (Stassun et al. 2019).

After this step, we are left with a sample of 197 Warm Jupiter candidates with 12 candidates on the preselected target list.

\subsection{TFOP WG Follow-up}

Several candidates that survived vetting have already been dispositioned as false positives (FPs) or false alarms (FAs) by the TESS Follow-up Observing Program (TFOP) ${ }^{50}$ Subgroup 1 (SG1) using seeing-limited photometry and Subgroup 2 (SG2) using recon spectroscopy. As ground-based follow-up observations progress, we will continue to drop newly discovered FPs and FAs from the catalog. Table 1 lists the 15 FPs and FA that survived our vetting and 15 FPs that were removed during the TCE vetting discussed in the previous subsection.

The SG1 follow-up observations attempt to (1) rule out or identify nearby eclipsing binaries (NEBs) as sources of the TESS data detection, (2) detect the transit-like event on-target to confirm the event depth and thus the TESS photometric deblending factor, (3) refine the TESS ephemeris, (4) provide additional epochs of transit center time measurements to supplement TTV analysis, and (5) place constraints on transit depth differences across optical filter bands. We used the TESS Transit Finder, which is a customized version of the Tapir software package (Jensen 2013), to schedule SG1 transit observations.

The SG2 follow-up observations attempt to provide spectroscopic parameters that will more precisely constrain the masses and radii of planet host stars, to detect false positives caused by spectroscopic binaries (SBs), and to identify stars unsuitable for precise RV measurements, such as rapid rotators.

Joint analysis of ground-based and TESS data for planet candidates in our catalog that have been verified to be on-target is beyond the scope of this work. The ground-based data will be presented in follow-up statistical validation or radial velocity confirmation publications. However, in the process of following up on these candidates, we identified several nearby eclipsing binaries (NEBs) that are the cause of their corresponding signals in the TESS data. We also identified a few

\footnotetext{
${ }^{50}$ https://tess.mit.edu/followup
} 
Table 1

False Positives (FPs) and False Alarms (FAs) identified by TFOP WG SG1 and SG2

\begin{tabular}{|c|c|c|c|}
\hline $\begin{array}{l}\text { TIC ID } \\
\text { (1) }\end{array}$ & $\begin{array}{l}\text { TOI name } \\
\text { (2) }\end{array}$ & $\begin{array}{c}\mathrm{SG} 1 / 2 \\
\text { Disposition } \\
\text { (3) }\end{array}$ & $\begin{array}{c}\text { Comments/Reference } \\
\text { (4) }\end{array}$ \\
\hline 207081058 & TOI-121.01 & SB1 & $\cdots$ \\
\hline 281710229 & TOI-308.01 & BEB & $\cdots$ \\
\hline 167418898 & TOI-383.01 & $\mathrm{NPC}, \mathrm{BD} / \mathrm{SB} 1$ & $\cdots$ \\
\hline 271900960 & TOI-389.01 & NEB & $\cdots$ \\
\hline 123482865 & TOI-569b & $\mathrm{BD}$ & Carmichael et al. (2020) \\
\hline 196286587 & TOI-592.01 & SB1 & $\cdots$ \\
\hline 101395259 & TOI-623.01 & SEB1 & $\ldots$ \\
\hline 293853437 & TOI-629.01 & $\mathrm{BD}$ & $\begin{array}{c}\text { Carmichael et al. (2021, in } \\
\text { preparation) }\end{array}$ \\
\hline 151681127 & TOI-671.01 & SB1 & $\cdots$ \\
\hline 151959065 & TOI-673.01 & SEB1 & $\cdots$ \\
\hline 308050066 & TOI-679.01 & SB1 & $\cdots$ \\
\hline 55383975 & TOI-694b & SB1 & Mireles et al. (2020) \\
\hline 309402106 & TOI-710.01 & SEB1 & $\ldots$ \\
\hline 131081852 & TOI-758.01 & SB2 & $\cdots$ \\
\hline 294780517 & TOI-792.01 & NEB & $\cdots$ \\
\hline 143526444 & TOI-803.01 & NEB & $\cdots$ \\
\hline 100757807 & TOI-811b & $\mathrm{BD}$ & Carmichael et al. (2021) \\
\hline 461271719 & TOI-838.01 & SB1 & $\ldots$ \\
\hline 216935214 & TOI-902.01 & SB2 & $\ldots$ \\
\hline 399144800 & TOI-1213.01 & SEB1 & $\ldots$ \\
\hline 231736113 & TOI-1406b & $\mathrm{BD}$ & Carmichael et al. (2020) \\
\hline 235067594 & $\cdots$ & NEB & $\begin{array}{c}\text { CTOI; Montalto et al. } \\
\text { (2020); actual star TIC- } \\
235067595\end{array}$ \\
\hline 23733479 & $\cdots$ & NEB & actual star TIC-23733473 \\
\hline 92833442 & $\cdots$ & NEB & actual star TIC-92833424 \\
\hline 140344868 & $\cdots$ & NEB & actual star TIC-140344846 \\
\hline 177350401 & $\cdots$ & NEB & actual star TIC-177350397 \\
\hline 308885493 & $\cdots$ & BEB & actual star TIC-308885490 \\
\hline 394662124 & $\cdots$ & NEB & actual star TIC-394662125 \\
\hline 418883593 & $\ldots$ & NEB & actual star TIC-414477523 \\
\hline 425170378 & $\cdots$ & FA & $\cdots$ \\
\hline
\end{tabular}

Notes. Photometric dispositions are abbreviated as follows. EB: eclipsing binary. BEB: blended EB. NEB: nearby EB. NPC: nearby planet candidate. FA: false alarm. Spectroscopic dispositions are abbreviated as follows. SB: spectroscopy binary. SB1: single-lined spectra showing RV variation too large to be caused by a planet. SEB1: SB1 with orbital solution. SB2: double-lined $\mathrm{SB}$ moving in phase with the photometric orbit. BD: brown dwarf.

systems with strong transit depth chromaticity, which we interpret as a blended eclipsing binary (BEB) in the follow-up photometric aperture. We also identified one candidate that is an FA after it was not detected on or off target in the groundbased follow-up data. Further analysis of the TESS data led us to conclude that the apparent signal in the TESS data is a detrending residual due to high scattered light near the beginning of the two corresponding TESS orbits. The followup light-curve data are available at ExoFOP-TESS. ${ }^{51}$ Table 2 lists observatories that have participated in SG1 follow-up observations of these targets.

\section{Light-curve Characterization}

TESS light curves are obtained from the MIT Quick Look Pipeline (QLP; Huang et al. 2019, 2020b, 2020c). To reduce

\footnotetext{
51 https://exofop.ipac.caltech.edu/tess
}

the computational effort for light-curve fitting, we trim the light curves around each transit to roughly six times the transit duration. We generally use the QLP detrended light curves (i.e., KSPSAP_FLUX), which are corrected for systematics using Kepler splines (Shallue \& Vanderburg 2018). However, we identify some KSPSAP_FLUX light curves showing overdetrended transit signals (e.g., transit signals being washed out due to the correction). In such cases, we switch to the simple aperture photometry (SAP) light curves. We also notice that transit signals could be masked by the TESS quality flag if they are close to momentum dumps. To avoid missing any transit data, we do not apply the TESS quality flag to produce the trimmed light curves. Instead, we manually remove truly abnormal data points (e.g., a sudden $10 \%$ flux deviation on a single data point) in the trimmed light curves.

For the preselected targets with 2 minute cadence data available, we obtain their TESS light curves processed with the Science Processing Operations Center (SPOC) pipeline (Jenkins et al. 2010). The SPOC pipeline is a descendant of the Kepler mission pipeline based at the NASA Ames Research Center (Jenkins et al. 2002, 2010), analyzing target pixel postage stamps that are obtained for preselected target stars. We download the publicly available data from the Mikulski Archive for Space Telescopes (MAST) using the lightkurve package (Lightkurve Collaboration et al. 2018). Similar to FFI light curves, we use PDCSAP light curves in most cases but switch to SAP light curves if the PDCSAP light curves are overdetrended.

\subsection{Light-curve Modeling}

We use a quadratic limb-darkening transit model (Mandel \& Agol 2002), along with a Gaussian process (GP) likelihood function, to infer planet properties from TESS light curves. The free parameters in our transit model are $\left\{\rho_{\text {circ }}, b, r_{\mathrm{p}} / r_{\star}, P, T_{0}, u_{0}, u_{1}\right\}$, where $\rho_{\text {circ }}$ is the stellar density assuming a circular orbit, $b$ is the impact parameter of the transiting planet, $r_{\mathrm{p}} / r_{\star}$ is the planet-star radius ratio, $P$ is the planet orbital period, $T_{0}$ is the mid-transit time of the first observed transit, and $u_{0}$ and $u_{1}$ are the quadratic limb darkening coefficients (Kipping 2013). We note that we fit our candidates' stellar densities, $\rho_{\text {circ }}$, assuming they have circular orbits. We later compare the marginalized $\rho_{\text {circ }}$ to $\rho_{\star}$, the "true" stellar densities derived from the isochrone fitting, to constrain the candidates' eccentricities (Section 3.2). To allow TTV characterization, we adopt a slightly different approach for candidates with 3 + transits. Instead of fitting the orbital period and mid-transit time, we fit mid-transit times for each transit (i.e., $T_{1 . . N}$ ). We take the orbital period as a fixed value in the fitting, and it is only involved in the computation of the transit duration. Since the orbital period is known with much greater precision than the precision with which the transit duration can be determined, and since the transit duration has a weak scaling with the orbital period $\left(\tau \propto P^{1 / 3}\right)$, fixing the orbital period for light-curve modeling and computing it later from a linear fit to mid-transit times does not significantly affect the inference on any of the parameters. Finally, to account for correlated noise in the light curves, we adopt a GP kernel including a diagonal jitter term $\left(s_{\mathrm{gp}}\right)$ to characterize the light-curve white noise and a Matern $3 / 2$ term $\left(\sigma_{\mathrm{gp}}\right.$ for the amplitude and $\rho_{\mathrm{gp}}$ for the timescale) to account for photometric variability (Foreman-Mackey et al. 2017; Foreman-Mackey 2018). In Table 3, we list the priors we put on the free parameters described above. For the preselected targets with both 2 minute and 30 minute cadence data, we perform a joint fit of parameters $\left\{\rho_{\text {circ }}, b, r_{\mathrm{p}} / r_{\star}, T_{1 . . N}, u_{0}, u_{1}\right\}$, but give 
Table 2

Facilities Used for SG1 Follow-up Observations

\begin{tabular}{|c|c|c|c|c|}
\hline Observatory & Location & $\begin{array}{l}\text { Aperture } \\
\text { (m) }\end{array}$ & $\begin{array}{l}\text { Pixel Scale } \\
\text { (arcseconds) }\end{array}$ & $\begin{array}{c}\text { FOV } \\
\text { (arcminutes) }\end{array}$ \\
\hline Antarctic Search for Transiting ExoPlanets (ASTEP) & Concordia Station, Antarctica & 0.4 & 0.93 & $63 \times 63$ \\
\hline Brierfield Private Observatory & Bellingen, New S. Wales, Australia & 0.36 & 1.47 & $50 \times 50$ \\
\hline Chilean-Hungarian Automated Telescope (CHAT) & Las Campanas Observatory, Chile & 0.7 & 0.6 & $21 \times 21$ \\
\hline Evans Private Telescope & El Sauce Observatory, Chile & 0.36 & 1.47 & $19 \times 13$ \\
\hline Hazelwood Private Observatory & Churchill, Victoria, Australia & 0.32 & 0.55 & $20 \times 14$ \\
\hline Hungarian Automated Tel. Network-South (HATS) & Chile/Namibia/Australia & 0.18 & 3.7 & $492 \times 492$ \\
\hline Las Cumbres Observatory Global Telescope (LCOGT) & Chile/South Africa/Australia & 1.0 & 0.39 & $26 \times 26$ \\
\hline MEarth-South & La Serena, Chile & 0.4 & 0.84 & $29 \times 29$ \\
\hline Perth Exoplanet Survey Telescope (PEST) & Perth, Australia & 0.3 & 1.2 & $31 \times 21$ \\
\hline TRAPPIST-North & Oukaimeden Observatory, Morocco & 0.6 & 0.60 & $20 \times 20$ \\
\hline TRAPPIST-South & La Silla, Chile & 0.6 & 0.64 & $22 \times 22$ \\
\hline Wide Angle Search for Planets-South (WASP-South) & Sutherland, South Africa & 0.1 & 13.7 & $468 \times 468$ \\
\hline
\end{tabular}

Note. TRAPPIST (Jehin et al. 2011).

Table 3

Summary of Priors Used for the Light-curve Fits

\begin{tabular}{llr}
\hline \hline Parameter & Description & Distribution \\
\hline Transit model & & $\log$ Uniform $\left(10^{-3}, 10^{3}\right)$ \\
$\rho_{\text {circ }}$ & Stellar density assuming a circular orbit $\left(\mathrm{g} \mathrm{cm}^{-3}\right)$ & Uniform $(-2,2)$ \\
$b$ & Impact parameter & $\log$ Uniform $($ testval-5, testval +5$)$ \\
$r_{\mathrm{p}} / r_{\star}$ & Planet-star radius ratio & Uniform $($ testval-5, testval +5$)$ or constant \\
$P$ & Orbital period (days) & Uniform $($ testval-5, testval +5$)$ \\
$T_{1 . . N}$ & Mid-transit times (BJD-2457000) & Adopted from Kipping $(2013)$ \\
$u_{0}, u_{1}$ & Quadratic limb-darkening coefficients & $\log$ Uniform $\left(30 \times 10^{-6}, 1\right)$ \\
\hline Gaussian process model & & $\log$ Uniform $\left(30 \times 10^{-6}, 1\right)$ \\
$s_{\mathrm{gp}}$ & Photometric jitter & $\operatorname{logUniform}(0.001,1000)$ \\
$\rho_{\mathrm{gp}}$ & Amplitude of the Matern 3/2 kernel &
\end{tabular}

Notes. testval: test values. The absolute value of the impact parameter is used when computing light curves.

each data set a different set of GP-kernel parameters to treat the difference on time sampling.

The light-curve fits use the exoplanet package (ForemanMackey et al. 2019) that implements the quadratic limb-darkening transit model using starry (Luger et al. 2019; Agol et al. 2020) and the Matern 3/2 GP model in celerite (Foreman-Mackey et al. 2017). The light-curve model is averaged into 30 minute bins over 15 evaluations per bin (i.e., texp $=0.02083$, oversample $=15$ ) for the 30 minute cadence light curves, and averaged into 2 minute bins over 8 evaluations per bin (i.e., texp $=0.00139$, oversample $=8$ ) for the 2 minute cadence light curves. For each set of light curves, we sample four chains using the Markov chain Monte Carlo (MCMC) technique with gradient-based proposals (Hoffman \& Gelman 2011; Neal 2012; Betancourt 2017). Each chain contains 5000 tuning steps and 3000 sampling steps. We set the target accept rate universally to 0.99 to reduce divergences caused by the degeneracy between $\rho_{\text {circ }}$ and $b$ for some $v$-shaped light curves. We assess MCMC convergence using the Gelman-Rubin diagnostic (i.e., $\hat{\mathcal{R}}<1.1$ for convergence), trace plots, and corner plots (ForemanMackey 2016) of the marginal joint distributions. We combine all four chains to get the posterior distribution. For a typical Warm Jupiter candidate with three transits (e.g., TOI-172b; Rodriguez et al. 2019), it takes $\sim 30$ minutes to analyze the light curves, benefiting from the efficiency of gradient-based proposals and No U-Turns (NUTs) sampling (Hoffman \& Gelman 2011).

\subsection{Post-light-curve Analysis}

Our light-curve fitting identifies a large group of Warm Jupiter candidates $(\sim 100)$ with grazing orbits that were not previously identified in Section 2. Their impact parameter posteriors have a mode centered around $b=1$ with large uncertainties (e.g., $\sigma_{b}=0.3-0.5$ ). We remove these candidates from our catalog because we cannot constrain their planet-star radius ratios, due to poorly constrained impact parameters. The range of possible radii extends to the stellar companion regime in many cases.

We assess TTVs for Warm Jupiter candidates with $3+$ transits. We calculate a best-fit linear ephemeris to the mid-transit times using the medians and uncertainties on midtransit time posteriors from light-curve fitting. We perform a least-squares fitting to

$$
T_{n}=T_{c}+n P
$$

where $T_{n}$ is the mid-transit time for the $n$th transit, $T_{c}$ is the conjunction time for reference, and $P$ is the orbital period of the planet. To compute the $O-C$ (observed-minus-calculated) times, we subtract the linear ephemeris $T_{n}$ from the observed 


\section{TESS Warm Jupiter Candidates (Southern Ecliptic Hemisphere)}

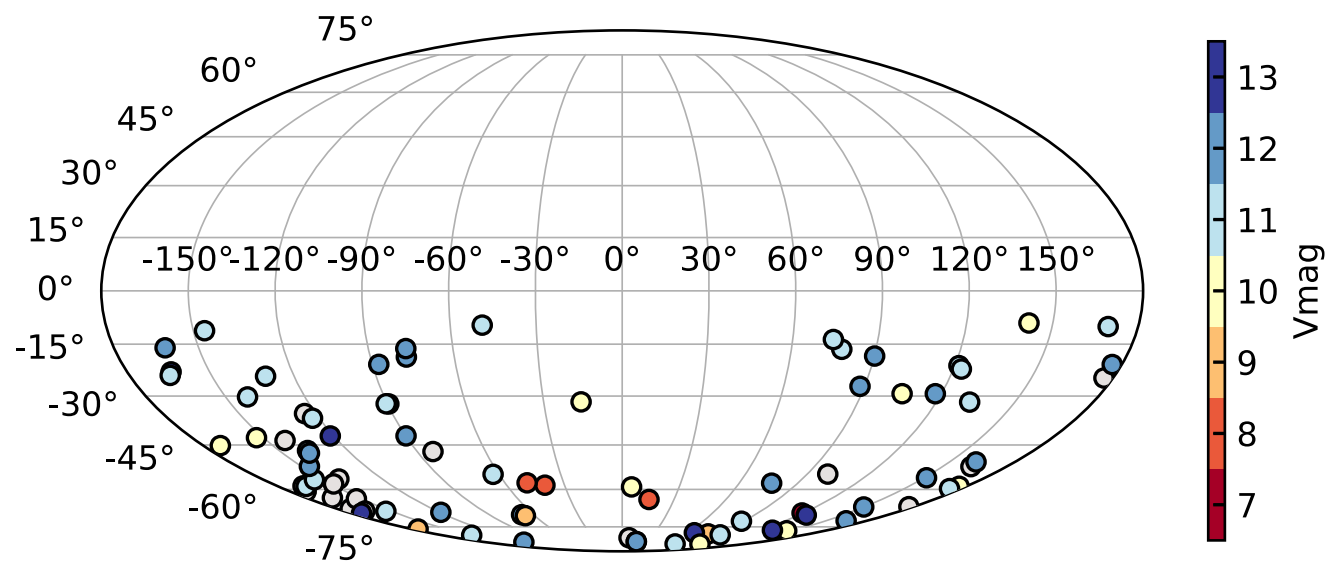

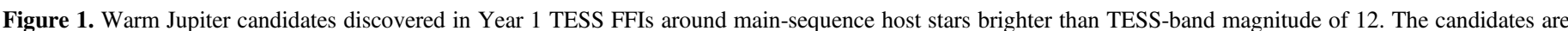

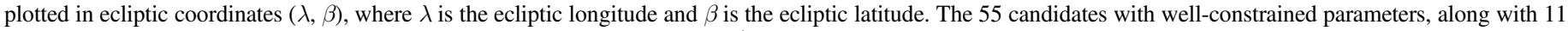

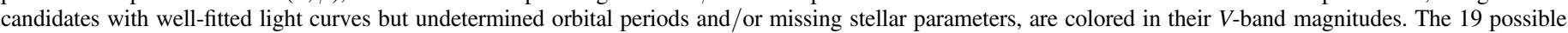
candidates with unconstrained impact parameters and radii are colored in gray. Most of our targets are bright enough for ground-based follow-up observations.

mid-transit times. To evaluate the significance of the TTV signal, we find the absolute difference between every pair of $O-C$ data points and normalize it by their uncertainties (i.e., the quadratic error in the two mid-transit times). For example, for a pair of mid-transit data points $T_{x}$ and $T_{y}$ with $O-C$ s of $(O-C)_{x}$ and $(O-C)_{y}$ and uncertainties of $\sigma_{T_{x}}$ and $\sigma_{T_{y}}$, respectively, their TTV significance $\sigma_{\mathrm{TTV} \mid x, y}$ is calculated as

$$
\sigma_{\mathrm{TTV} \mid x, y}=\frac{\left|(O-C)_{x}-(O-C)_{y}\right|}{\sqrt{\sigma_{T_{x}}^{2}+\sigma_{T_{y}}^{2}}} .
$$

We take the maximum value of the TTV significance of every pair of the mid-transit times as the significance level of the TTV detection of the system.

The orbital eccentricity of a planet can be inferred from its transit light curve, sometimes termed the "photoeccentric" effect, although the solution is degenerate with the planet's argument of periapse. Using the posterior distribution of $\rho_{\text {circ }}$ from light-curve fitting and the posterior distribution of $\rho_{\star}$ from the isochrone fitting, we compute a joint posterior distribution for $(e, \omega)$ following Dawson \& Johnson (2012).

\section{Candidate Catalog}

Our vetting process results in a catalog of 55 Warm Jupiter candidates in the FFIs in Year 1 of the TESS Prime Mission data. Due to the depth of our survey (i.e., Tmag $<12$ ), 19 of our candidates have not yet been identified as TOIs. We present their light curves in Appendix A. A complete figure set (19 figures) of the FFI light curves of non-TOI candidates is available in the online journal. In Section 4.1, we tabulate our candidates with their host star and planet properties and present their TTV and eccentricity analysis. In Section 4.2, we introduce 11 additional Warm Jupiter candidates with undetermined orbital periods and/ or missing stellar parameters, and we list 19 more possible Warm Jupiter candidates with unconstrained impact parameters.

\subsection{A Catalog of Warm Jupiter Candidates}

We present 55 Warm Jupiter candidates discovered in TESS Sectors 1-13 in FFIs around main-sequence host stars brighter than TESS magnitude of 12. In Figure 1, we display our candidates in ecliptic coordinates and color them according to their $V$-band magnitude. In Table 4, we tabulate the 55 candidates with their planet and host star properties. A detailed description of each column can be found in the table caption. The 19 candidates that were not originally released as TOIs by the TESS team can be identified from the "TOI Name" column (Column 2) with no TOI names listed. Some of the non-TOI targets have been independently vetted by other groups (e.g., Montalto et al. 2020) and reported as Community TOIs (CTOIs), labeled in Column 17.

In Figure 2, we present the TTV analysis for the 35 Warm Jupiter systems with $3+$ transits. In each panel, we show the $O-C$ diagram (i.e., the observed mid-transit times with the calculated mid-transit times subtracted) of the planet candidates in the system. The panels are sorted by the TTV significance. About half of the systems are observed in multiple TESS sectors. One of the most prominent TTV systems is TOI-216 (TIC-55652896; Dawson et al. 2019; Kipping et al. 2019; Dawson et al. 2021), with an outer Warm Jupiter (labeled as open circles) in 2:1 meanmotion resonance with the inner Warm Neptune (labeled as black dots). The system has a TTV significance level of 27.1 using our metric discussed in Section 3.2. We do not identify any other systems with TTV signals as significant as TOI-216's in the rest of the catalog. TOI-1130 (TIC-254113311; Huang et al. 2020a) is another TESS multi-transiting-planet system with an outer 8.4 day Warm Jupiter (TOI-1130c) and an inner 4.1 day hot Neptune (TOI-1130b). Our TTV analysis on the system shows that TOI1130c (i.e., black dots) has no obvious TTV signals, and TOI1130 b (i.e., open circles) has some tentative TTV signals. We might be able to detect the TTV signals of TOI-1130c when combined with the TESS Extended Mission data. Meanwhile, the system demonstrates that, due to the short observing baseline, even a target with a nearby companion can still show no significant TTV signals.

As shown in Figure 2, we evaluate the significance of the TTV signals (i.e., $>3 \sigma, 2-3 \sigma$, and $<2 \sigma$ ) for each system. We find that $4 / 35(\sim 11 \%)$ systems show a $>3 \sigma$ TTV detection, colored and labeled in lavender; $4 / 36(\sim 11 \%)$ candidates show $2-3 \sigma$ TTV detection, colored and labeled in green; and 27/35 $(77 \%)$ systems show less than $2 \sigma$ TTV signals, colored and 
Table 4

(Continued)

\begin{tabular}{|c|c|c|c|c|c|c|c|c|c|c|c|c|c|c|c|c|}
\hline $\begin{array}{l}\text { TIC ID } \\
\text { (1) }\end{array}$ & $\begin{array}{l}\text { TOI } \\
\text { (2) }\end{array}$ & $\begin{array}{c}R_{\star} \\
\left(\mathrm{R}_{\odot}\right) \\
(3)\end{array}$ & $\begin{array}{l}\rho_{\star} \\
\left(\rho_{\odot}\right) \\
(4)\end{array}$ & $\begin{array}{l}\rho_{\text {circ }} \\
\left(\rho_{\odot}\right) \\
(5)\end{array}$ & $\begin{array}{l}\delta \\
(\mathrm{ppt}) \\
(6)\end{array}$ & $\begin{array}{c}R_{\mathrm{p}} \\
\left(R_{\oplus}\right) \\
(7)\end{array}$ & $\begin{array}{l}b \\
\text { (8) }\end{array}$ & $\begin{array}{c}P \\
\text { (day) } \\
(9)\end{array}$ & $\begin{array}{c}T_{c} \\
\text { (BJD-2457000) } \\
(10)\end{array}$ & $\begin{array}{l}e \\
(11)\end{array}$ & $\begin{array}{l}\omega \\
\left({ }^{\circ}\right) \\
(12)\end{array}$ & $\begin{array}{l}e_{\mathrm{RV}} \\
(13)\end{array}$ & $\begin{array}{l}\omega_{\mathrm{RV}} \\
\left({ }^{\circ}\right) \\
(14)\end{array}$ & $\begin{array}{l}\sigma_{\mathrm{TTV}} \\
(15)\end{array}$ & $\begin{array}{l}\text { TSM } \\
\text { (16) }\end{array}$ & $\begin{array}{c}\text { Other Name/Reference } \\
\text { (17) }\end{array}$ \\
\hline 254113311 & $1130 \mathrm{c}$ & $0.695_{-0.036}^{+0.036}$ & $2.195_{-0.265}^{+0.265}$ & $1.920_{-0.250}^{+0.478}$ & $35.686_{-16.610}^{+76.608}$ & $14.292_{-3.810}^{+11.245}$ & $0.986_{-0.091}^{+0.182}$ & $8.351_{-0.000}^{+0.000}$ & $1649.547_{-0.001}^{+0.001}$ & $0.073_{-0.073}^{+0.22}$ & $\begin{array}{l}{[-13,62],} \\
{[113,195]} \\
{[-51,12],} \\
{[168,230]}\end{array}$ & $0.047_{-0.027}^{0.04}$ & $332_{-55}^{+24}$ & 1.1 & 100 & Huang et al. (2020a) \\
\hline 409794137 & $1478 \mathrm{~b}$ & $0.988_{-0.044}^{+0.044}$ & $0.974_{-0.126}^{+0.126}$ & $1.116_{-0.333}^{+0.200}$ & $8.944_{-0.358}^{+0.502}$ & $10.213_{-0.509}^{+0.525}$ & $0.339_{-0.225}^{+0.218}$ & $10.181_{-0.000}^{+0.000}$ & $1485.738_{-0.001}^{+0.001}$ & $0.085_{-0.085}^{+0.23}$ & $\begin{array}{l}{[-44,30],} \\
{[153,224]}\end{array}$ & $0.024_{-0.017}^{+0.032}$ & $250_{-130}^{+120}$ & 1.0 & 33 & Rodriguez et al. (2021) \\
\hline 308098254 & 1906.01 & $1.113_{-0.048}^{+0.048}$ & $0.701_{-0.090}^{+0.090}$ & $0.619_{-0.269}^{+0.260}$ & $8.517_{-0.613}^{+0.903}$ & $11.249_{-0.648}^{+0.710}$ & $0.494_{-0.318}^{+0.214}$ & $9.627_{-0.000}^{+0.000}$ & $1538.718_{-0.000}^{+0.000}$ & $0.087_{-0.083}^{+0.28}$ & $\begin{array}{l}{[-62,12],} \\
{[168,241]}\end{array}$ & $0.43_{-0.005}^{+0.005}$ & $358_{-2}^{+2}$ & 0.1 & 15 & $\begin{array}{l}\text { WASP-162b; Hellier } \\
\text { et al. (2019) }\end{array}$ \\
\hline 190271688 & 1963.01 & $1.062_{-0.054}^{+0.054}$ & $0.776_{-0.107}^{+0.107}$ & $0.814_{-0.073}^{+0.097}$ & $21.059_{-1.058}^{+0.077}$ & $16.751_{-0.922}^{+0.922}$ & $0.659_{-0.051}^{+0.036}$ & $12.636_{-0.001}^{+0.001}$ & $1607.245_{-0.000}^{+0.000}$ & $0.055_{-0.055}^{+0.22}$ & $\begin{array}{l}{[-35,26],} \\
{[152,213]}\end{array}$ & $\ldots$ & $\ldots$ & & 57 & - \\
\hline 371188886 & 2000.01 & $1.074_{-0.053}^{+0.053}$ & $0.769_{-0.104}^{+0.104}$ & $1.221_{-0.544}^{+0.298}$ & $3.730_{-0.200}^{+0.258}$ & $7.160_{-0.405}^{+0.433}$ & $0.389_{-0.261}^{+0.273}$ & $9.127_{-0.001}^{+0.001}$ & $1535.058_{-0.003}^{+0.003}$ & $0.19_{-0.17}^{+0.2}$ & $\begin{array}{l}{[-41,51],} \\
{[137,221]}\end{array}$ & $\ldots$ & $\ldots$ & 2.3 & 16 & - \\
\hline 147660886 & 2005.01 & $2.103_{-0.077}^{+0.077}$ & $0.161_{-0.021}^{+0.021}$ & $1.394_{-0.559}^{+0.314}$ & $2.498_{-0.149}^{+0.164}$ & $11.461_{-0.546}^{+0.578}$ & $0.366_{-0.250}^{+0.257}$ & $17.306_{-0.002}^{+0.002}$ & $1549.835_{-0.002}^{+0.002}$ & $0.65_{-0.15}^{+0.14}$ & {$[29,148]$} & $\ldots$ & $\ldots$ & & 13 & - \\
\hline 394287035 & 2328.01 & $0.829_{-0.042}^{+0.042}$ & $1.429_{-0.167}^{+0.167}$ & $2.693_{-0.937}^{+0.508}$ & $9.130_{-0.538}^{+0.598}$ & $8.639_{-0.504}^{+0.519}$ & $0.327_{-0.219}^{+0.254}$ & $17.102_{-0.000}^{+0.000}$ & $1313.386_{-0.000}^{+0.000}$ & $0.24_{-0.22}^{+0.18}$ & $\begin{array}{l}{[-27,52],} \\
{[123,209]}\end{array}$ & $\ldots$ & $\ldots$ & 0.1 & 14 & СТOI \\
\hline 24358417 & 2338.01 & $1.028_{-0.047}^{+0.047}$ & $0.881_{-0.125}^{+0.125}$ & $3.631_{-1.020}^{0.592}$ & $10.028_{-0.547}^{+0.508}$ & $11.218_{-0.595}^{+0.600}$ & $0.278_{-0.191}^{+0.230}$ & $22.652_{-0.003}^{+0.003}$ & $1481.407_{-0.002}^{+0.002}$ & $0.48_{-0.15}^{+0.2}$ & $\begin{array}{l}{[20,55]} \\
{[68,170]}\end{array}$ & $\ldots$ & $\ldots$ & $\cdots$ & 11 & - \\
\hline 279514271 & 2339.01 & $2.166_{-0.096}^{+0.096}$ & $0.086_{-0.015}^{+0.015}$ & $0.085_{-0.059}^{+0.050}$ & $2.032_{-0.239}^{+0.526}$ & $10.720_{-0.862}^{+1.334}$ & $0.528_{-0.355}^{+0.297}$ & $9.774_{-0.000}^{+0.000}$ & $1351.802_{-0.008}^{+0.008}$ & $0.13_{-0.11}^{+0.29}$ & $\begin{array}{l}{[-64,29],} \\
{[152,243]}\end{array}$ & $\ldots$ & $\ldots$ & 1.2 & 1 & - \\
\hline 204671232 & 2361.01 & $0.984_{-0.045}^{+0.045}$ & $0.971_{-0.125}^{+0.125}$ & $1.001_{-0.230}^{+0.134}$ & $10.749_{-0.539}^{+0.520}$ & $11.114_{-0.565}^{+0.572}$ & $0.259_{-0.177}^{+0.212}$ & $8.716_{-0.003}^{+0.003}$ & $1569.062_{-0.007}^{+0.007}$ & $0.062_{-0.062}^{+0.24}$ & $\begin{array}{l}{[-47,19],} \\
{[160,228]}\end{array}$ & $\cdots$ & $\cdots$ & 2.3 & 15 & $\begin{array}{l}\text { CTOI; Montalto et al. } \\
\text { (2020) }\end{array}$ \\
\hline 238542895 & $\cdots$ & $0.896_{-0.030}^{+0.030}$ & $1.204_{-0.137}^{+0.137}$ & $0.730_{-0.136}^{+0.076}$ & $10.233_{-0.432}^{+0.431}$ & $9.872_{-0.391}^{+0.398}$ & $0.229_{-0.157}^{+0.203}$ & $8.385_{-0.000}^{+0.000}$ & $1485.880_{-0.001}^{+0.001}$ & $0.21_{-0.094}^{+0.27}$ & $\begin{array}{l}{[-66,-24],} \\
{[203,247]}\end{array}$ & $\ldots$ & $\ldots$ & 1.6 & 18 & CTOI \\
\hline 282498590 & $\cdots$ & $1.051_{-0.145}^{+0.145}$ & $0.778_{-0.279}^{+0.279}$ & $0.556_{-0.172}^{+0.101}$ & $10.780_{-0.543}^{+0.656}$ & $11.896_{-1.603}^{+1.674}$ & $0.343_{-0.232}^{+0.231}$ & $10.095_{-0.002}^{+0.002}$ & $1474.825_{-0.001}^{+0.001}$ & $0.16_{-0.14}^{+0.27}$ & $\begin{array}{l}{[-66,-8],} \\
{[189,246]}\end{array}$ & $\ldots$ & $\ldots$ & $\cdots$ & 22 & $\begin{array}{l}\text { CTOI; Montalto et al. } \\
\text { (2020) }\end{array}$ \\
\hline 290403522 & $\ldots$ & $1.384_{-0.052}^{+0.052}$ & $0.435_{-0.067}^{+0.067}$ & $0.059_{-0.007}^{+0.010}$ & $6.596_{-0.246}^{+0.231}$ & $12.247_{-0.499}^{+0.512}$ & $0.538_{-0.092}^{+0.057}$ & $22.375_{-0.000}^{+0.000}$ & $1324.188_{-0.003}^{+0.003}$ & $0.61_{-0.076}^{+0.16}$ & $\begin{array}{l}{[-86,-52],} \\
{[232,266]}\end{array}$ & $\ldots$ & $\ldots$ & 1.5 & 18 & СТОI \\
\hline 380836882 & $\ldots$ & $1.197_{-0.053}^{+0.053}$ & $0.574_{-0.087}^{+0.087}$ & $0.392_{-0.102}^{+0.052}$ & $6.541_{-0.302}^{+0.367}$ & $10.573_{-0.534}^{+0.553}$ & $0.282_{-0.195}^{+0.219}$ & $8.012_{-0.000}^{+0.000}$ & $1652.826_{-0.001}^{+0.001}$ & $0.17_{-0.11}^{+0.28}$ & $\begin{array}{l}{[-65,-17],} \\
{[197,243]}\end{array}$ & $\ldots$ & $\ldots$ & 0.3 & 18 & CTOI \\
\hline 382200986 & $\ldots$ & $0.680_{-0.036}^{+0.036}$ & $2.182_{-0.232}^{+0.232}$ & $2.002_{-0.449}^{+0.251}$ & $8.810_{-0.346}^{+0.417}$ & $6.975_{-0.394}^{+0.399}$ & $0.279_{-0.191}^{+0.202}$ & $92.498_{-0.005}^{+0.005}$ & $1243.912_{-0.010}^{+0.010}$ & $0.06_{-0.06}^{+0.25}$ & $\begin{array}{l}{[-51,1]} \\
{[175,233]}\end{array}$ & $\ldots$ & $\ldots$ & 3.7 & 5 & $\begin{array}{l}\text { CTOI; Montalto et al. } \\
\text { (2020) }\end{array}$ \\
\hline 357202877 & & $1.116_{-0.044}^{+0.044}$ & $0.645_{-0.084}^{+0.084}$ & $1.143_{-0.549}^{+0.693}$ & $14.388_{-2.518}^{+3.440}$ & $14.643_{-1.468}^{+1.728}$ & $0.570_{-0.372}^{+0.223}$ & $9.555_{-0.001}^{+0.001}$ & $1534.787_{-0.003}^{+0.003}$ & $0.1_{-0.084}^{+0.33}$ & $\begin{array}{l}{[-35,56],} \\
{[132,213]}\end{array}$ & & & 1.0 & 20 & - \\
\hline 254142310 & $\ldots$ & $1.633_{-0.074}^{+0.074}$ & $0.231_{-0.045}^{+0.045}$ & $0.264_{-0.123}^{+0.080}$ & $2.986_{-0.252}^{+0.322}$ & $9.743_{-0.616}^{+0.675}$ & $0.414_{-0.279}^{+0.267}$ & $9.311_{-0.002}^{+0.002}$ & $1652.052_{-0.004}^{+0.004}$ & $0.11_{-0.099}^{+0.27}$ & $\begin{array}{l}{[-57,27],} \\
{[152,236]}\end{array}$ & & & 0.6 & 8 & - \\
\hline 395113305 & $\cdots$ & $0.886_{-0.043}^{+0.043}$ & $1.272_{-0.162}^{+0.162}$ & $0.284_{-0.159}^{+0.150}$ & $7.345_{-0.917}^{+1.252}$ & $8.294_{-0.667}^{+0.0787}$ & $0.506_{-0.338}^{+0.259}$ & $9.963_{-0.008}^{+0.008}$ & $1607.420_{-0.006}^{+0.006}$ & $0.48_{-0.1}^{+0.27}$ & $\begin{array}{l}{[-82,-45],} \\
{[224,262]}\end{array}$ & & & $\ldots$ & 11 & - \\
\hline 180989820 & $\ldots$ & $1.256_{-0.058}^{+0.058}$ & $0.544_{-0.088}^{+0.088}$ & $0.084_{-0.026}^{+0.017}$ & $4.508_{-0.236}^{+0.338}$ & $9.223_{-0.484}^{+0.524}$ & $0.356_{-0.243}^{+0.216}$ & $9.873_{-0.001}^{+0.001}$ & $1511.287_{-0.004}^{+0.004}$ & $0.63_{-0.1}^{+0.14}$ & $\begin{array}{l}{[-86,-52],} \\
{[233,265]}\end{array}$ & $\ldots$ & $\ldots$ & 1.4 & 12 & - \\
\hline 4598935 & & $1.042_{-0.040}^{+0.040}$ & $0.801_{-0.108}^{+0.108}$ & $0.460_{-0.231}^{+0.159}$ & $3.515_{-0.367}^{0.475}$ & $6.744_{-0.455}^{+0.525}$ & $0.438_{-0.299}^{+0.271}$ & $24.663_{-0.007}^{+0.007}$ & $1539.451_{-0.006}^{+0.006}$ & $0.19_{-0.12}^{+0.35}$ & $\begin{array}{l}{[-71,-19],} \\
{[202,248]}\end{array}$ & & & & 5 & - \\
\hline 464300749 & $\ldots$ & $1.678_{-0.056}^{+0.056}$ & $0.270_{-0.044}^{+0.044}$ & $4.896_{-2.229}^{+1.439}$ & $4.007_{-0.316}^{0.334}$ & $11.589_{-0.601}^{+0.620}$ & $0.385_{-0.265}^{+0.281}$ & $18.098_{-0.003}^{+0.003}$ & $1529.321_{-0.006}^{+0.006}$ & $0.78_{-0.13}^{+0.098}$ & {$[34,143]$} & $\ldots$ & $\ldots$ & 1.1 & 11 & - \\
\hline 7536985 & $\cdots$ & $1.908_{-0.086}^{+0.086}$ & $0.163_{-0.036}^{+0.036}$ & $0.315_{-0.133}^{+0.091}$ & $7.271_{-0.503}^{+0.557}$ & $17.745_{-1.015}^{+1.074}$ & $0.397_{-0.268}^{+0.253}$ & $12.732_{-0.005}^{+0.005}$ & $1502.907_{-0.004}^{+0.004}$ & $0.25_{-0.22}^{+0.19}$ & $\begin{array}{l}{[-32,54],} \\
{[128,210]}\end{array}$ & $\ldots$ & $\cdots$ & $\cdots$ & 8 & - \\
\hline 76228620 & $\cdots$ & $1.194_{-0.051}^{+0.051}$ & $0.571_{-0.090}^{+0.090}$ & $0.343_{-0.217}^{+0.227}$ & $4.492_{-0.626}^{+1.118}$ & $8.768_{-0.768}^{+1.045}$ & $0.567_{-0.381}^{+0.254}$ & $9.779_{-0.002}^{+0.002}$ & $1645.400_{-0.004}^{+0.004}$ & $0.078_{-0.075}^{+0.37}$ & $\begin{array}{l}{[-71,-4],} \\
{[186,253]}\end{array}$ & $\ldots$ & $\ldots$ & 0.5 & 11 & - \\
\hline
\end{tabular}




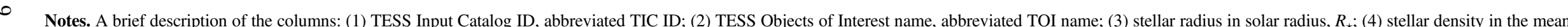

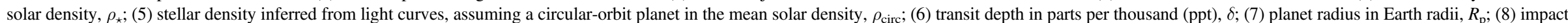

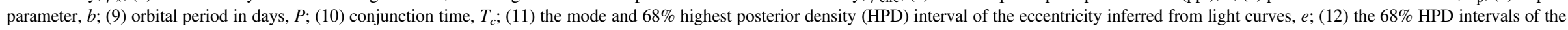

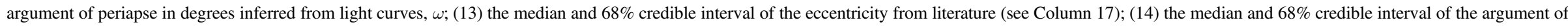

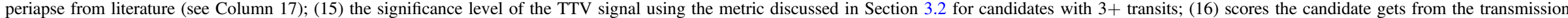

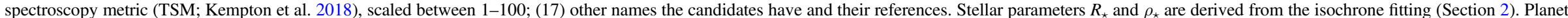

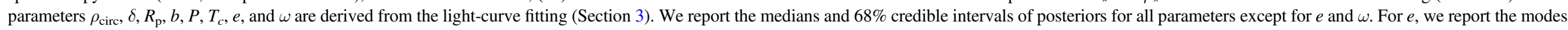
and $68 \%$ highest posterior density (HPD) intervals. For $\omega$, we report the 68\% HPD intervals to take account of multimodal distributions.

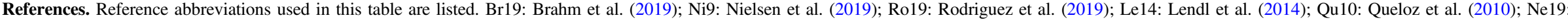

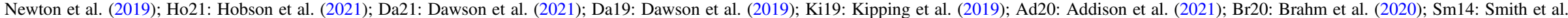
(2014); Jo20: Jordán et al. (2020); He17: Hellier et al. (2017); Hu20: Huang et al. (2020a); He19: Hellier et al. (2019). 

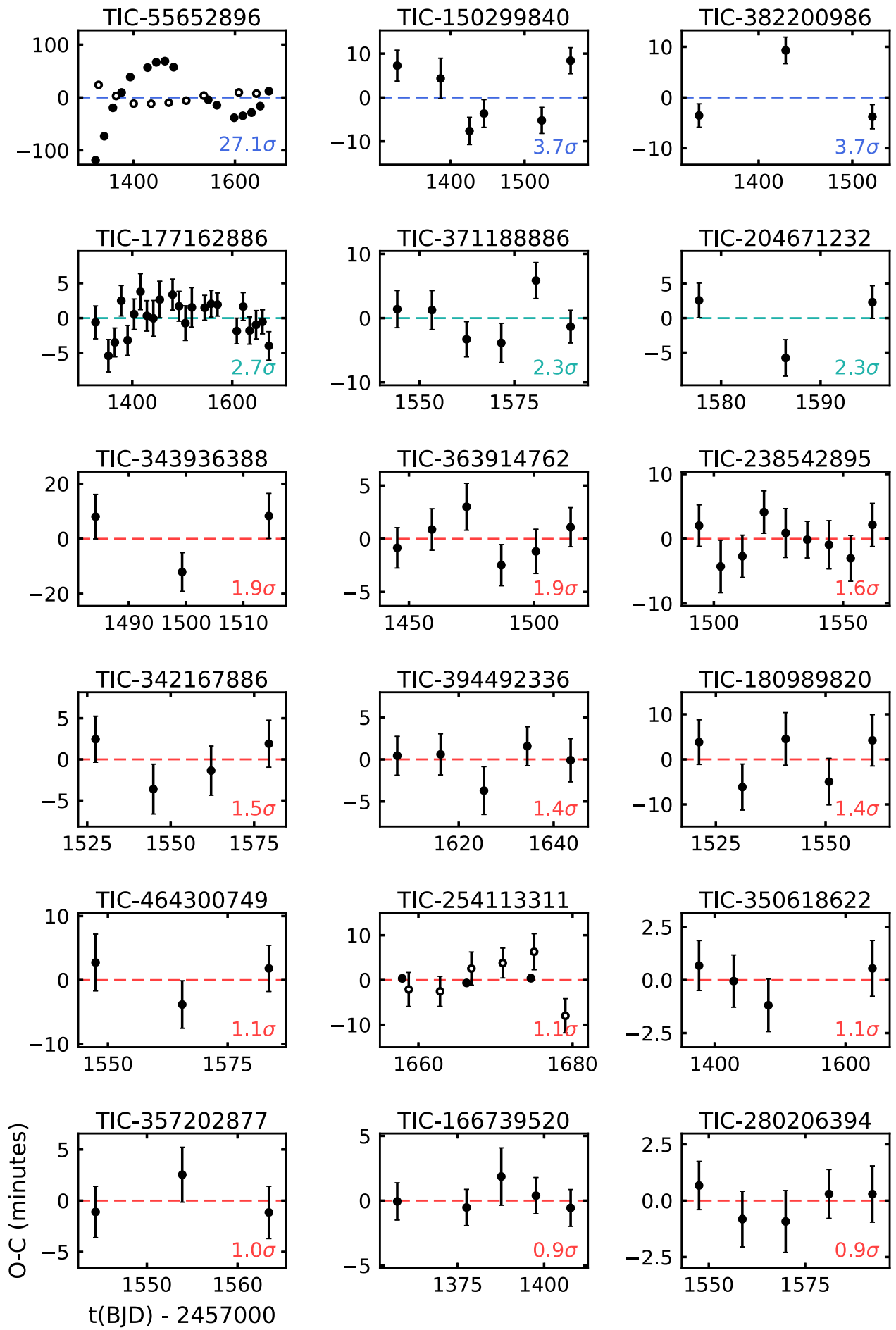
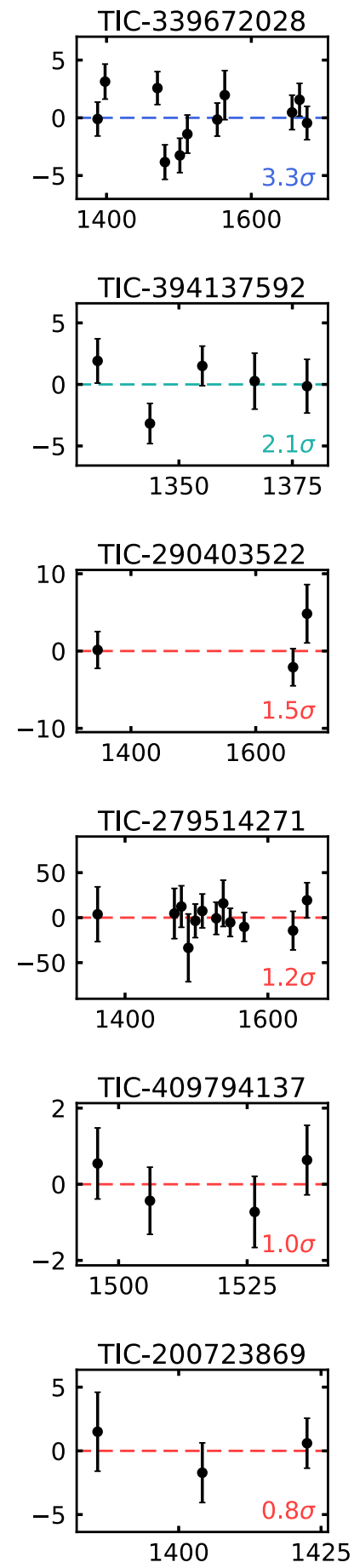

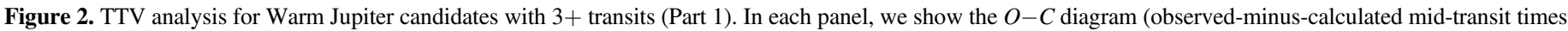

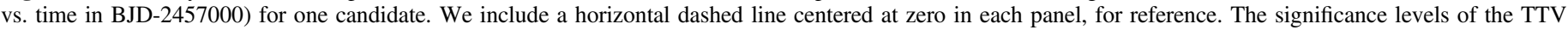

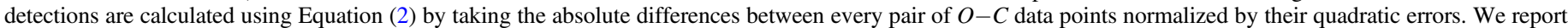

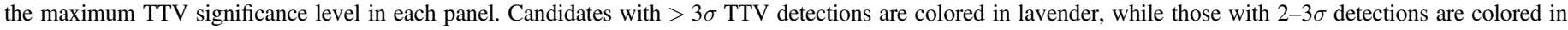

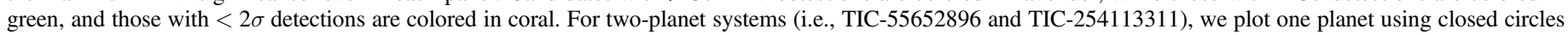
and the other using open circles.

labeled in coral. The horizontal dashed line in each panel is centered at zero, for reference. Although they do not have the strongest TTV detections, a few systems show TTV patterns that could be sinusoidal, e.g.,

1. TIC-150299840

2. TIC-382200986

\section{TIC-371188886 \\ 4. TIC-343936388,}

which might be worthwhile to explore further. Many of the systems (i.e., systems colored in coral) show no significant TTV signals. The lack of detected TTVs does not rule out the presence of other planets and may be due to the lack of 

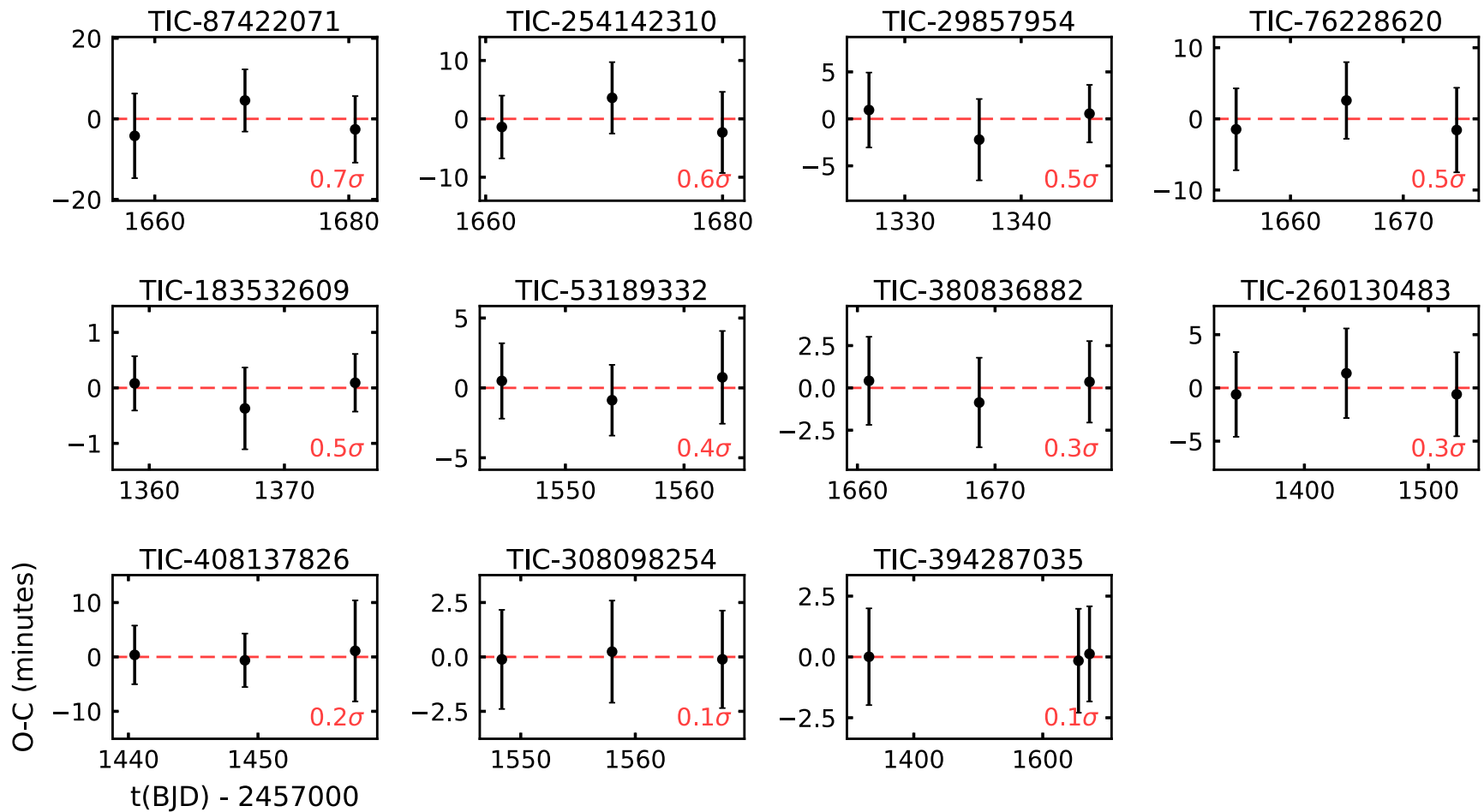

Figure 2. (Continued.)

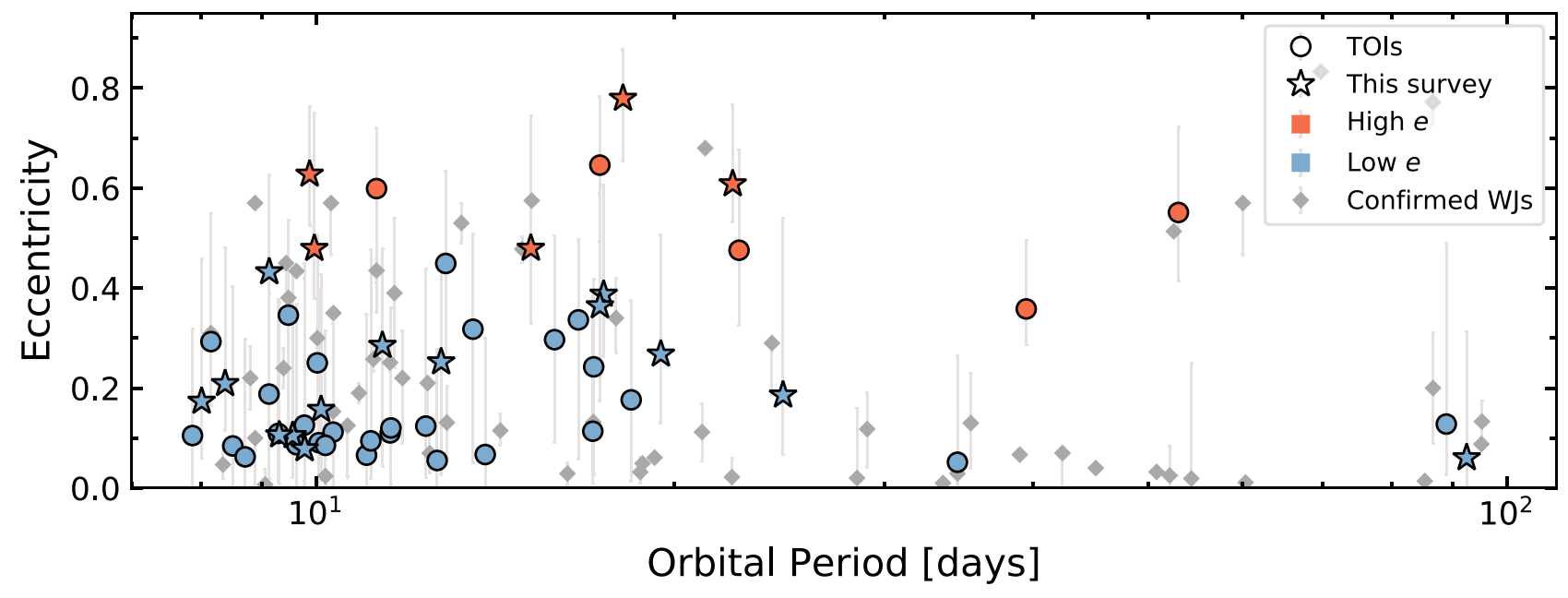

Figure 3. Eccentricity vs. orbital period of the 55 Warm Jupiter candidates discovered in Year 1 TESS FFIs around host stars brighter than Tmag of 12 . The eccentricities are inferred from the candidates' stellar densities and presented by their posterior modes and the 68\% highest posterior density (HPD) intervals. TOIs are labeled with circles, and unique targets yield from our survey are labeled with stars. If the lower bound of the 95\% HPD interval of the eccentricity is greater than 0.2 , we identify the planet as a high-e planet and have it colored in orange; otherwise, we have it colored in blue. We have 10 candidates identified as high- $e$ planets. All 79 confirmed (as of March 2021 from NASA's Exoplanet Archive) Warm Jupiters, defined here as planets larger than 6 Earth radii with orbital periods of 8-200 days, are plotted in gray dots.

precision of mid-transit times (i.e., demonstrated by error bars in Figure 2) and/or short observing baselines. With the TESS Extended Mission observation, which will provide a longer observing baseline and finer cadence data, we expect to improve the TTV analysis for many of the Warm Jupiter systems.

We characterize the eccentricities of 55 Warm Jupiter candidates using the "photoeccentric" effect discussed in Section 3.2. Given the asymmetrical distribution of the eccentricity posteriors and the bimodal distribution of the argument-of-periapse posteriors, we report their modes and the $68 \%$ highest posterior density (HPD) intervals instead of the medians and $68 \%$ quantiles. In Figure 3, we present the eccentricity-versus-orbital period of these candidates. Each candidate is labeled by the mode of its eccentricity posterior, and the gray error bar indicates the 68\% HPD. As shown in Figure 3, we identify a collection of Warm Jupiter candidates that are possibly on highly elliptical orbits and have them colored in orange. To identify these high- $e$ candidates, we use the criterion of whether the lower bound of the 95\% HPD of the eccentricity posterior is greater than 0.2 . The criterion here is not driven by theoretical models, but rather is intended to 


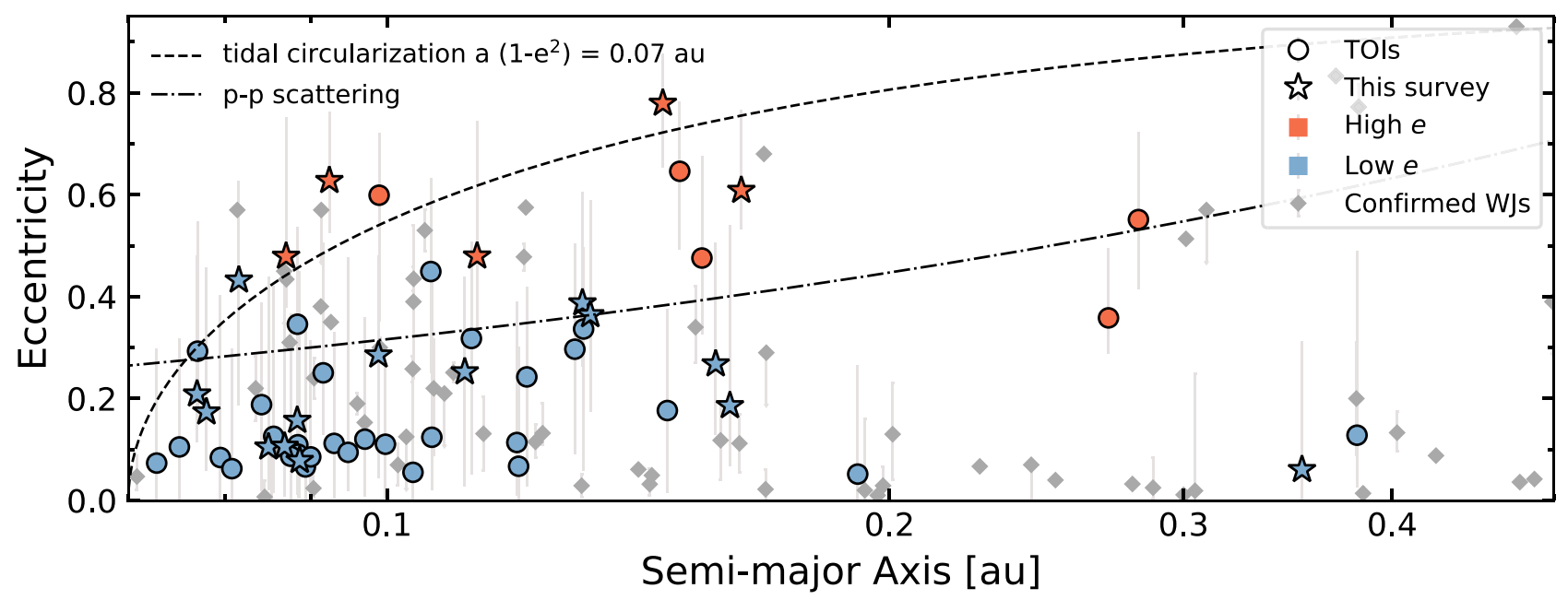

Figure 4. Eccentricity vs. semimajor axis of the 55 Warm Jupiter candidates discovered in Year 1 TESS FFIs around host stars brighter than Tmag of 12. The eccentricities are inferred from the candidates' stellar densities and presented by their posterior modes and the 68\% highest posterior density (HPD) intervals. We color a collection of candidates that are possibly on highly elliptical orbits in orange, according to their eccentricities and HPD intervals (similar to Figure 3). We present two reference curves to demonstrate possible Warm Jupiter origin channels. The tidal circularization line (i.e., the dashed line; $a_{\text {final }}=a\left(1-e^{2}\right)=0.07$ au) illustrates one possible formation pathway of a Warm Jupiter under high-e migration. Above the line, the planet's orbit will shrink to a semimajor axis of 0.07 au or smaller. The planet-planet scattering line (i.e., the dotted-dashed line) demonstrates the approximate maximum eccentricity to which a planet could be excited by nearby companions without undergoing collisions and having its eccentricity damped. The line can be understood as the maximum eccentricity one would expect for multiple Warm Jupiters formed in situ.

prioritize a list of targets for ground-based follow-up observations. The 10 targets are:

1. TIC-350618622 (TOI-201.01; Hobson et al. 2021)

2. TIC-130415266 (TOI-588.01)

3. TIC-280206394 (TOI-677b; Jordán et al. 2020)

4. TIC-147660886 (TOI-2005.01)

5. TIC-24358417 (TOI-2338.01)

6. TIC-290403522

7. TIC-395113305

8. TIC-180989820

9. TIC-464300749

10. TIC-343936388.

In Figure 4, we plot the eccentricity versus the semimajor axis of the candidates, to demonstrate possible Warm Jupiter formation pathways. If Warm Jupiters are formed at large semimajor axes and migrate inward via high-eccentricity tidal migration, they will follow a tidal circularization track of constant angular momentum, such as the dashed line shown in Figure 4. During the process of the high- $e$ migration, the planet loses orbital energy $\left(E_{\mathrm{p}}=-G M_{\star} m_{\mathrm{p}} / 2 a\right)$, due to the tidal dissipation in the planet, and thus shrinks its orbit. The angular momentum $\left(L_{\mathrm{p}}=m_{\mathrm{p}} \sqrt{G M_{\star} a\left(1-e^{2}\right)}\right)$, however, stays roughly the same. As a result, the planet reduces its eccentricity as its semimajor axis gets smaller, following an evolution track of $a_{\text {final }}=a\left(1-e^{2}\right)$, where $a_{\text {final }}$ is a constant and the semimajor axis the planet ends up with by the end of the migration (considering tidal dissipation in the planet only). The tidal circularization timescale has a strong dependence on $a_{\text {final }}$ (i.e., $\tau \propto a_{\text {final }}^{8}$; Eggleton et al. 1998). Generally, planets with $a_{\text {final }}<0.05$ au are likely to get circularized in a star's lifetime, whereas planets with $a_{\text {final }}>0.1$ au are unlikely to do so. Because of the uncertainty in the tidal dissipation efficiency and thus on the tidal circularization timescale, some of our shortest-period, circular-orbit warm Jupiters could have have undergone complete tidal circularization. The dashed line in Figure 4 has a final semimajor axis of $0.07 \mathrm{au}$, an illustrative value for the critical final semimajor axis. Warm Jupiters on and above the dashed line are experiencing high-e migration and will have their final orbits shrunk to semimajor axes of 0.07 au or smaller. Planets below the dashed line could also experience high-e migration if they are coupled to outer companions and undergo eccentricity oscillations (Socrates et al. 2012; see Jackson et al. 2019 for a case study and Kane \& Raymond 2014 for an investigation of secular oscillations in radial-velocity discovered giant planet systems). If Warm Jupiters are instead formed in situ or arrived via disk migration, we expect to observe them at low eccentricities. The dotteddashed line in Figure 4 presents the approximate maximum eccentricity to which a planet could be excited via in situ planet-planet scattering (Petrovich et al. 2014; Equation (10) in Dawson \& Johnson 2018; Anderson et al. 2020). We assume a mass of $0.5 M_{\text {Jup }}$ and a radius of $2 R_{\text {Jup }}$ as illustrative values for young planets. Planets below the dotted-dashed line are consistent with in situ formation and disk migration. Planets that have undergone disk migration are expected to have small eccentricities (i.e., comparable to their disk scale heights; Duffel \& Chiang 2015) or at most moderate eccentricities $(e<0.4)$ if they are migrating in a disk cavity (Debras et al. 2021). However, Anderson \& Lai (2017) pointed out Warm Jupiters formed with low eccentricities could have their eccentricities excited by outer companions via secular interactions. In such scenarios, Warm Jupiters formed in situ or via disk migration can be observed above the dotted-dashed line.

We investigate possible correlations between planets' radii and eccentricities, as shown in Figure 5. We find the majority of Warm Jupiter candidates on highly elliptical orbits have a size between 8 and 13 Earth radii (for reference, Jupiter is $\sim 11$ $\left.R_{\oplus}\right)$. For both small (i.e., $\left.R_{p}<8 R_{\oplus}\right)$ and large $\left(R_{p}>12 R_{\oplus}\right)$ planets, we identify a lack of high-e planets. One possible explanation of the lack of high-e planets for the large planets $\left(R_{p}>12 R_{\oplus}\right)$ is that they are inflated by some heating sources, such as tidal heating from the planetary tidal dissipation (e.g., Bodenheimer et al. 2001), stellar heating from the stellar irradiation (e.g., Guillot \& Showman 2002), and thermal tides 


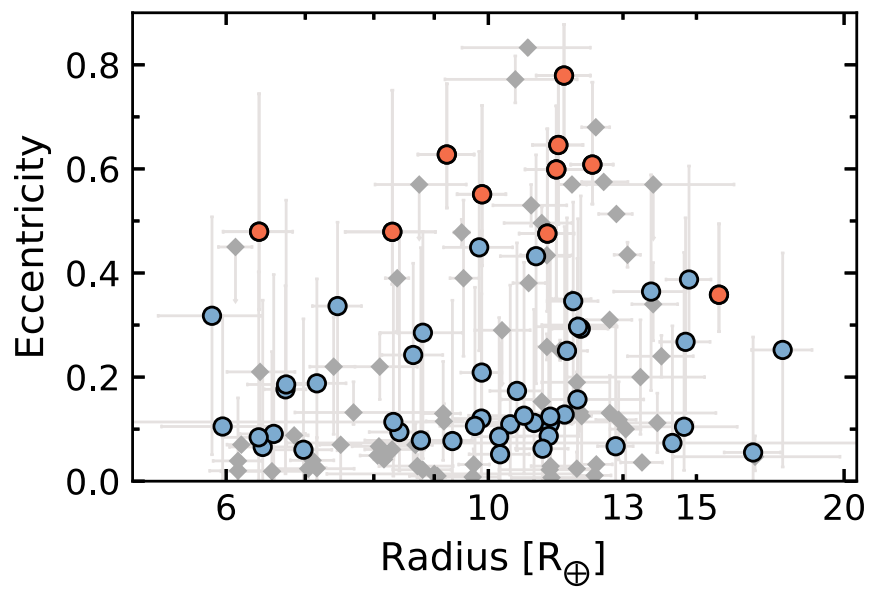

Figure 5. Eccentricity vs. planet radius in Earth radii for the 55 Warm Jupiter candidates. The eccentricities are inferred from the candidates' stellar densities and presented by their posterior modes and the $68 \%$ highest posterior density (HPD) intervals. The candidates are categorized into a high- $e$ population colored in orange and a low-e population colored in blue, given their eccentricities and HPD intervals (similar to Figures 4 and 5). The majority of the high- $e$ candidates (colored in orange) have a planetary size of 8-13 Earth radii.

caused by asynchronous rotation and orbital eccentricity (e.g., Arras \& Socrates 2010), and then tidally circularized by their host stars. However, all these theories require a small periapse of the planet, whereas many of our large Warm Jupiters have long orbital periods $(P>8$ days; some even have $P>15$ days $)$ and low eccentricities. Other possibilities are that the stellar radii could have errors unaccounted for in the uncertainties, or the large planets could instead be low-mass stars that might have a different dynamical history and/or circularization distance. For the small planets (i.e., $R_{p}<8 R_{\oplus}$ ), without a proper mass measurement, we cannot tell whether or not they are giant planets or superpuff planets. If they are superpuff planets instead, the lack of high- $e$ planets could be explained by a different origin channel(s) for low-mass planets. Our observations could suffer from small number statistics, since only $6 / 55$ candidates have a mode eccentricity greater than 0.5 . Moreover, astrophysical false positives, e.g., eclipse binaries and brown dwarfs, could contaminate our sample of Warm Jupiters and compromise our interpretations. More investigation, especially mass measurements of small and large planets, is required to draw any firm conclusions about the apparent correlation with planet size.

\subsection{Additional Candidates}

In Table 5, we introduce 11 additional Warm Jupiter candidates that are not included (i.e., Table 4) due to unconstrained ephemerides and eccentricities. The missing information is itemized in Table 5. Eight of these candidates have unconstrained orbital periods due to observation gaps in the TESS data. Ground-based follow-up or the TESS Extended Mission are likely to resolve their orbital period degeneracy. Four of them have missing stellar parameters and/or Gaia parallax, according to the TIC-v8 catalog. The missing stellar parameters lead to unconstrained planet eccentricity.

In addition, we list 19 more possible Warm Jupiter candidates that are not included due to their impact parameters and thus planet-star radius ratios being poorly constrained. The transit duration of these candidates is short and their light
Table 5

Warm Jupiter Candidates with Missing Information

\begin{tabular}{lcc}
\hline \hline TIC ID & TOI name & Missing Information \\
\hline 149601557 & TOI-1033.01 & $\sigma_{T_{\mathrm{eff}}}$ \\
296863792 & $\ldots$ & $T_{\mathrm{eff}}, \sigma_{T_{\mathrm{eff}}}, \mathrm{Gmag}, \sigma_{\mathrm{Gmag}}$ \\
306919690 & $\ldots$ & $T_{\mathrm{eff}}, \sigma_{T_{\mathrm{eff}}, \mathrm{plx}, \sigma_{\mathrm{plx}}}$ \\
270341214 & TOI-173.01 & 14 possible orbital periods \\
262746281 & TOI-603.01 & $P: 8.09$ or 16.18 days $^{\mathrm{a}}$ \\
308994098 & TOI-790.01 & $P: 99.77$ or 199.55 days \\
437329044 & TOI-1982.01 & $P: 8.58$ or 17.16 days \\
39218269 & TOI-2366.01 & $P: 8.60$ or 17.19 days \\
99133239 & $\ldots$ & $P: 9.17$ or 18.34 days \\
398466662 & $\ldots$ & $P: 8.77$ or 17.54 days \\
412635642 & $\ldots$ & $\sigma_{T_{\mathrm{eff}}}, \mathrm{plx}, \sigma_{\mathrm{plx}}, P: 8.71$ or 17.42 days \\
\hline
\end{tabular}

Notes. Gmag and plx stand for Gaia DR2 apparent $G$ magnitude and parallax. $P$ stands for orbital period, which is unconstrained due to observation gaps.

${ }^{\text {a }}$ Unique orbital period is later determined by ground-based follow-up observations.

curves are usually $v$-shaped. The posterior distributions of the impact parameter show a flat distribution between 0 and 1 , with a long tail above 1 . Because of the existence of the $b>1$ solution, planet radii could approach the substellar object regime for these candidates. We do not include these candidates in our catalog, to minimize false positives. Still, we list their TIC IDs here:
1. 4588737
2. 38815574
3. 76761591
4. 117817010
5. 304418238
6. 305345992
7. 359271114
8. 359732062
9. 379717270
10. 381982417
11. 384164973
12. 410395660
13. 412484721
14. 460205581 (TOI-837.01; Bouma et al. 2020)
15. 461968320
16. 462162948 (TOI-684.01)
17. 464124454
18. 467807015
19. 467807116 .

Finer-cadence photometric observations (e.g., from the TESS Extended Mission) may improve the constraints on the impact parameters and make some of the candidates qualified for the catalog.

\section{The Eccentricity Distribution}

Different origin channels (i.e., in situ formation, disk/high- $e$ migration) make different predictions for Warm Jupiters' eccentricities. To shed light on which one or more origin channels predominantly contribute to the Warm Jupiter population, we characterize the eccentricity distribution of our catalog using hierarchical Bayesian modeling (HBM). The philosophy of HBM is that each Warm Jupiter is a member of a specific population, and that members of each population share properties in common. Consequently, individual members 
reflect the properties of the population, and the population helps to make better inferences of the properties of individual members. Studying warm Jupiters as a catalog both provides information on the eccentricity distribution and improves the inference of the eccentricity of a single planet.

Transit durations can serve as proxies for the eccentricities of transiting planets and can be used to infer a population-wide eccentricity distribution under the assumption of a uniform distribution of impact parameters (e.g., as performed by studies such as Moorhead et al. (2011) and Kane et al. (2012) for Kepler planet candidates). For planets with large $\mathrm{S} / \mathrm{N}$ transits, transit shapes are well-resolved and we may constrain the impact parameters and their $\rho_{\text {circ }}$ assuming circular orbits. With both wellconstrained $\rho_{\text {circ }}$ and $\rho_{\star}$ (the true stellar density), we can constrain the planets' individual eccentricities using the "photoeccentric" effect (Kipping 2014a). For example, Dawson et al. (2015) applied the approach to search for super-eccentric Warm Jupiters in the Kepler sample, and Van Eylen et al. (2019) used the approach to constrain the eccentricity distribution of small Kepler planets. Here, we apply the method to a large sample of TESS Warm Jupiters. We note one caveat of this study is that, although we have removed several targets that have been dispositioned as FPs by TFOP groups, many targets in our sample are still planet candidates that have not yet been confirmed. The contamination of astrophysical FPs could compromise our interpretation of Warm Jupiters' eccentricity distribution.

We examine three functional forms of the eccentricity distribution, including a beta distribution, a Rayleigh distribution, and a mixture distribution with two Gaussian components. Both the beta distribution and the Rayleigh distribution have been broadly used in exoplanet eccentricity distribution studies, e.g., by Kipping (2013) on all radial-velocity discovered planets, by Fabrycky et al. (2014) and He et al. (2019) on Kepler systems, and by Shabram et al. (2016) on short-period Kepler planet candidates. The beta distribution is known for its flexibility in shape and is bounded in $[0,1]$, which can be conveniently adopted for the eccentricity distribution. The Rayleigh distribution is motivated by the planet-planet scattering origin of Warm Jupiters. Ida \& Makino (1992) find the Rayleigh distribution is a good descriptor for the eccentricity distribution generated by planet-planet scattering. We also introduce the two-component mixture distribution, inspired by the low- $e$ and high- $e$ populations predicted by Warm Jupiter's origin theories. The disk migration and in situ origin channels predict Warm Jupiters on circular or moderately elliptical orbits, whereas the high-e tidal migration origin channel predicts a group of Warm Jupiters on highly elliptical orbits. The two-component model is flexible enough to learn the fractions and expected eccentricities of the low-e and high-e populations of the sample.

We first introduce our HBM framework using the beta distribution. The frameworks for the Rayleigh and mixture distributions are similar except with minor changes on hyperparameters. Using the beta distribution as the functional form of the eccentricity distribution, we build the hierarchical model by introducing two hyperparameters, $\alpha_{e}$, and $\beta_{e}$. The probability distribution follows

$$
p\left(e \mid \alpha_{e}, \beta_{e}\right)=e^{\alpha_{e}-1}(1-e)^{\beta_{e}-1} / B\left(\alpha_{e}, \beta_{e}\right),
$$

where $B\left(\alpha_{e}, \beta_{e}\right)=\Gamma\left(\alpha_{e}\right) \Gamma\left(\beta_{e}\right) / \Gamma\left(\alpha_{e}+\beta_{e}\right)$ and $\Gamma$ is the gamma function. In Figure 6 , we display the Bayesian model using a directed factor graph. As shown in Figure 6, the hyperparameters $\alpha_{e}$ and $\beta_{e}$ are outside the plate. Inside the plate, we have $N$ planets

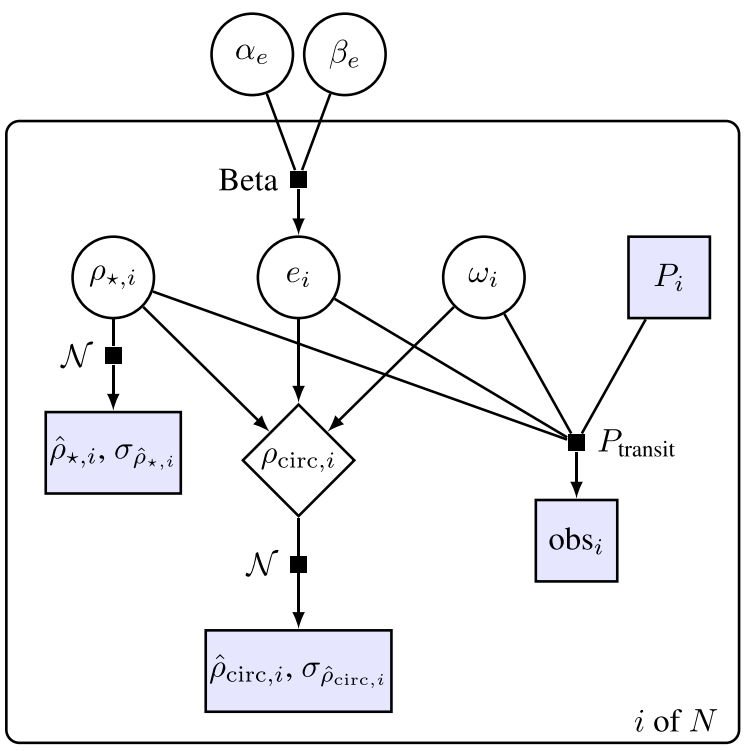

Figure 6. Graphic model of the hierarchical Bayesian model using the beta distribution as the functional form of the eccentricity distribution. Outside the plate, we have hyperparameters, $\alpha_{e}$ and $\beta_{e}$, describing the eccentricity distribution of the Warm Jupiter population. Inside the plate, we have individual parameters for each of the $N$ planets. Each planet $i$ has parameters, $\rho_{\star, i}, e_{i}$, and $\omega_{i}$, to be inferred from the model, labeled in circles. Here, $\rho_{\text {circ }, i}$ is a deterministic parameter that can be directly calculated from $\rho_{\star, i}, e_{i}$, and $\omega_{i}$, and is labeled in a diamond. The observed parameters, $\hat{\rho}_{\text {circ }, i}, \sigma_{\hat{\rho}_{\text {circ }, i},} \hat{\rho}_{\star, i}, \sigma_{\hat{\rho}_{\star}, i}$, and $\mathrm{obs}_{i}$, are labeled in lavender boxes. The planet orbital period, $P_{i}$, is an input parameter and taken as a constant in the model, similar to the other observed parameters.

and each planet has one set of the parameters listed in the graph. For each Warm Jupiter, we have two sets of observed parameters from previous analysis, the posterior distributions of stellar densities from isochrone fitting $\rho_{\star}$, and the posterior distributions of stellar densities from light-curve fitting $\rho_{\text {circ }}$, assuming all planets have circular orbits. The stellar density posteriors and the transit probabilities are observed parameters, colored in lavender and labeled in boxes in Figure 6. We approximate the stellar density posterior distributions as normal distributions, to simplify the model and reduce the computational effort. To relieve the concern that some stellar density posteriors are not nearly Gaussian, we compare the inferred eccentricities (and argument of periapsis) of individual targets using Gaussian-approximated posteriors to the inferred eccentricities using the "true" posteriors (e.g., Ford \& Rasio 2008; Kipping et al. 2012; see Dawson \& Johnson 2012 for a detailed description of the method). The values inferred using two types of posteriors are in good consistency with differences well below $1 \sigma$ uncertainties. The planet's orbital period $P_{i}$ is also taken as an observed parameter in the model, to calculate the transit probability. The relationship between $\rho_{\text {circ }, i}$ and $\rho_{\star, i}, e_{i}$, and $\omega_{i}$ is deterministic, so we label it using a diamond. We write down the posterior distribution of our hierarchical model as

$$
\begin{aligned}
p(\boldsymbol{\theta}, \boldsymbol{\beta} \mid \boldsymbol{X}) \propto p(\boldsymbol{X} \mid \boldsymbol{\theta}, \boldsymbol{\beta}) p(\boldsymbol{\theta} \mid \boldsymbol{\beta}) p(\boldsymbol{\beta}) \\
\propto \prod_{i=1}^{N}\left\{p\left(\hat{\rho}_{\mathrm{circ}, i}, \sigma_{\hat{\rho}_{\mathrm{cir}, i}} \mid \rho_{\mathrm{circ}, i}\right) p\left(\hat{\rho}_{\star, i}, \sigma_{\hat{\rho}_{\star, i}} \mid \rho_{\star, i}\right)\right. \\
\left.\quad p\left(\operatorname{obs}_{i} \mid \rho_{\star, i}, e_{i}, \omega_{i}, P_{i}\right)\right\} \\
\quad \times \prod_{i=1}^{N}\left\{p\left(e_{i} \mid \alpha_{e}, \beta_{e}\right) p\left(\rho_{\star, i}\right) p\left(\omega_{i}\right)\right\} \\
\quad \times p\left(\alpha_{e}\right) p\left(\beta_{e}\right),
\end{aligned}
$$


where $\boldsymbol{X}=\left\{\hat{\rho}_{\text {circ }, i}, \sigma_{\hat{\rho}_{\text {circ }},}, \hat{\rho}_{\star, i}, \sigma_{\hat{\rho}_{\star},}, \mathrm{obs}_{i}\right\}$ for the observed parameters, $\boldsymbol{\theta}=\left\{\rho_{\text {circ }, i}, \rho_{\star, i}, e_{i}, \omega_{i}\right\}$ for the individual parameters of each planet, and $\boldsymbol{\beta}=\left\{\alpha_{e}, \beta_{e}\right\}$ for the hyperparameters of the population. In the model, $P_{\text {transit }}$ describes the transit probability of a planet given its $\rho_{\star, i}, e_{i}, \omega_{i}$, and $P_{i}$. The planet's orbital period $P_{i}$ is taken from the light-curve modeling as a fixed value. Here, we list the probability distributions that we assume for the hierarchical Bayesian model:

$$
\begin{aligned}
& p\left(\hat{\rho}_{\text {circ }, i}, \sigma_{\hat{\rho}_{\text {cir }, i}} \mid \rho_{\text {circ }, i}\right) \sim \mathcal{N}\left(\rho_{\text {circ }, i} \mid \hat{\rho}_{\text {circ }, i}, \sigma_{\hat{\rho}_{\text {cir }, ~}^{2} i}\right) \\
& p\left(\hat{\rho}_{\star, i}, \sigma_{\hat{\rho}_{\star x}} \mid \rho_{\star, i}\right) \sim \mathcal{N}\left(\rho_{\star, i} \mid \hat{\rho}_{\star, i}, \sigma_{\hat{\rho}_{\star, i}}^{2}\right) \\
& p\left(\operatorname{obs}_{i} \mid \rho_{\star, i}, e_{i}, \omega_{i}, P_{i}\right) \sim \begin{cases}\frac{1}{\rho_{\star, i}^{1 / 3} P_{i}^{2 / 3}} \frac{1+e_{i} \sin \omega_{i}}{1-e_{i}^{2}} & \text { if } e_{i}<e_{\max , i} \\
0 & \text { if } e_{i} \geqslant e_{\max , i}\end{cases} \\
& p\left(e_{i} \mid \alpha_{e}, \beta_{e}\right) \sim \mathcal{B} \operatorname{Beta}\left(e_{i} \mid \alpha_{e}, \beta_{e}\right) \\
& p\left(\rho_{\star, i}\right) \sim \mathcal{N}\left(\hat{\rho}_{\star, i}, \sigma_{\hat{\rho}_{\star, i}}^{2}\right) \\
& p\left(\omega_{i}\right) \sim \operatorname{Uniform}(0,2 \pi) \\
& p\left(\alpha_{e}\right) \sim \operatorname{Uniform}(0,10) \\
& p\left(\beta_{e}\right) \sim \operatorname{Uniform}(0,10),
\end{aligned}
$$

where $\mathcal{N}$ indicates a normal distribution. The $e_{\max , i}$ is the maximum eccentricity a planet can reach without getting tidally disrupted (i.e., $\left.e_{\max , i}=1-R_{\star, i} / a_{i}\right)$. The $p\left(\operatorname{obs}_{i} \mid \rho_{\star, i}, e_{i}, \omega_{i}, P_{i}\right)$ is the transit probability to correct the observation biases in the eccentricities of transiting planets (Burke 2008; Equation (9) in Winn 2010; Kipping 2014b).

Two more functional forms of the eccentricity distribution are examined: the Rayleigh distribution and the mixture distribution. The Rayleigh distribution can be written as

$$
p\left(e \mid \sigma_{e}\right)=\frac{e}{\sigma_{e}} \exp \left(-e^{2} / 2 \sigma_{e}^{2}\right)
$$

where $\sigma_{e}$ is a free hyperparameter. ${ }^{52}$ We use $p\left(\sigma_{e}\right) \sim$ $\operatorname{Uniform}(0,10 / \sqrt{2})$ as the hyperprior for $\sigma_{e}$. For the mixture distribution, we use two Gaussian distributions to describe the low-e and high-e populations of Warm Jupiters, respectively. The probability distribution can be written as

$$
\begin{aligned}
& p\left(e \mid f_{1}, f_{2}, \mu_{1}, \mu_{2}, \sigma_{1}, \sigma_{2}\right) \\
& \quad=f_{1} \mathcal{N}\left(e \mid \mu_{1}, \sigma_{1}^{2}\right)+f_{2} \mathcal{N}\left(e \mid \mu_{2}, \sigma_{2}^{2}\right),
\end{aligned}
$$

where $f_{1}$ and $f_{2}$ present the fractions of two distributions and have a sum of 1 . For both the Rayleigh distribution and the mixture distribution, we bound the eccentricity distribution between 0 and 1 . In the mixture model, we require both $f_{1}$ and $f_{2}$ to be greater than 0.05 , to avoid divergence of the model. We restrict $\mu_{1}$ and $\mu_{2}$ to be ordered, to avoid swaps between modes (i.e., label switching). A full description of the hyperpriors in

\footnotetext{
52 The Rayleigh distribution is a special form of the Weibull distribution, $f(e \mid \alpha, \beta)=\alpha e^{\alpha-1} \exp \left(-(e / \beta)^{\alpha}\right) / \beta^{\alpha}$, where $\alpha=2$ and $\beta=\sqrt{2} \sigma_{e}$.
}

Table 6

Summary of the Posteriors of the Hyperparameters for Hierarchical Bayesian Models

\begin{tabular}{lccc}
\hline \hline Distribution & \multicolumn{3}{c}{ Hyperparameter Posteriors } \\
\hline Beta & $\alpha_{e}$ & $\beta_{e}$ & \\
& $1.776_{-0.771}^{+1.385}$ & $4.082_{-1.634}^{+2.647}$ & \\
\hline Rayleigh & $\sigma_{e}$ & & \\
& $0.259_{-0.029}^{+0.032}$ & & $\sigma_{1}$ \\
\hline Mixture & $f_{1}$ & $\mu_{1}$ & $0.059_{-0.043}^{+0.072}$ \\
& $0.530_{-0.209}^{+0.227}$ & $0.174_{-0.088}^{+0.114}$ & $\sigma_{2}$ \\
& $f_{2}$ & $\mu_{2}$ & $0.135_{-0.071}^{+0.063}$ \\
& $0.470_{-0.227}^{+0.209}$ & $0.484_{-0.095}^{+0.107}$ & \\
\hline
\end{tabular}

Note. We report the medians and $68 \%$ credible intervals of the posteriors.

the mixture model is shown below:

$$
\begin{aligned}
p\left(f_{1}\right) & \sim \text { Uniform }(0,1) \\
p\left(f_{2}\right) & =1-f_{1} \\
p\left(\mu_{1}\right), p\left(\mu_{2}\right) & \sim \text { Uniform }(0,1) \\
p\left(\sigma_{1}\right), p\left(\sigma_{2}\right) & \sim \text { Half Cauchy }(1) \\
p\left(\text { prior } 1 \mid f_{1}, f_{2}\right) & = \begin{cases}0 & \text { if } f_{1}, f_{2}<0.05 \\
1 & \text { otherwise }\end{cases} \\
p\left(\text { prior2 } \mid \mu_{1}, \mu_{2}\right) & = \begin{cases}0 & \text { if } \mu_{1}>\mu_{2} \\
1 & \text { otherwise }\end{cases} \\
p\left(\text { prior3 } \mid \sigma_{1}, \sigma_{2}\right) & = \begin{cases}0 & \text { if } \sigma_{1}, \sigma_{2} \notin[0,1] \\
1 & \text { otherwise }\end{cases} \\
p\left(\text { prior4 } \mid \mu_{1}, \sigma_{1}\right) & =\operatorname{CDF}\left[\mathcal{N}\left(\mu_{1}, \sigma_{1}^{2}\right)=1, \mathcal{N}\left(\mu_{1}, \sigma_{1}^{2}\right)=0\right] \\
p\left(\text { prior5 } \mid \mu_{2}, \sigma_{2}\right) & =\operatorname{CDF}\left[\mathcal{N}\left(\mu_{2}, \sigma_{2}^{2}\right)=1, \mathcal{N}\left(\mu_{2}, \sigma_{2}^{2}\right)=0\right],
\end{aligned}
$$

where $\operatorname{CDF}[a, b]=\operatorname{CDF}[a]-\operatorname{CDF}[b]$. Prior 1 prevents the fraction of either Gaussian component from being zero, to cause divergence. Prior 2 sets $\mu_{1}$ and $\mu_{2}$ to be ordered. Prior 3 bounds $\sigma_{1}$ and $\sigma_{2}$ between 0 and 1. Last, priors 4 and 5 bound the eccentricity distribution between 0 and 1 .

We build the hierarchical Bayesian models and sample posteriors using PyMC3 (Salvatier et al. 2016) ${ }^{53}$ We sample four parallel chains, each chain with 40,000 tuning steps and 10,000 draws. A target accept rate of 0.99 is used to avoid divergences. The MCMC convergence is evaluated by the Gelman-Rubin diagnostic (i.e., $\hat{\mathcal{R}}<1.1$ for convergence), trace plots, and corner plots (Foreman-Mackey 2016) of the marginal joint distributions. The posterior distributions and covariances of the hyperparameters for the beta distribution and the mixture model can be found in Figures B1 and B2. A summary of best-fit models is shown in Table 6 .

The eccentricity distributions assuming the three functional forms discussed above are shown in Figure 7. In each panel, we plot the best-fitting model as a dark line by calculating the distribution using the medians of the posteriors of the hyperparameters, along with 500 draws from the posteriors. Compared to the Rayleigh distribution, the beta distribution infers lower eccentricities for the population. A similar distinction was also found by Shabram et al. (2016) when the authors did a comparison of the beta and Rayleigh distribution

\footnotetext{
$\overline{53}$ All codes used in this project are available from J.D. upon request.
} 


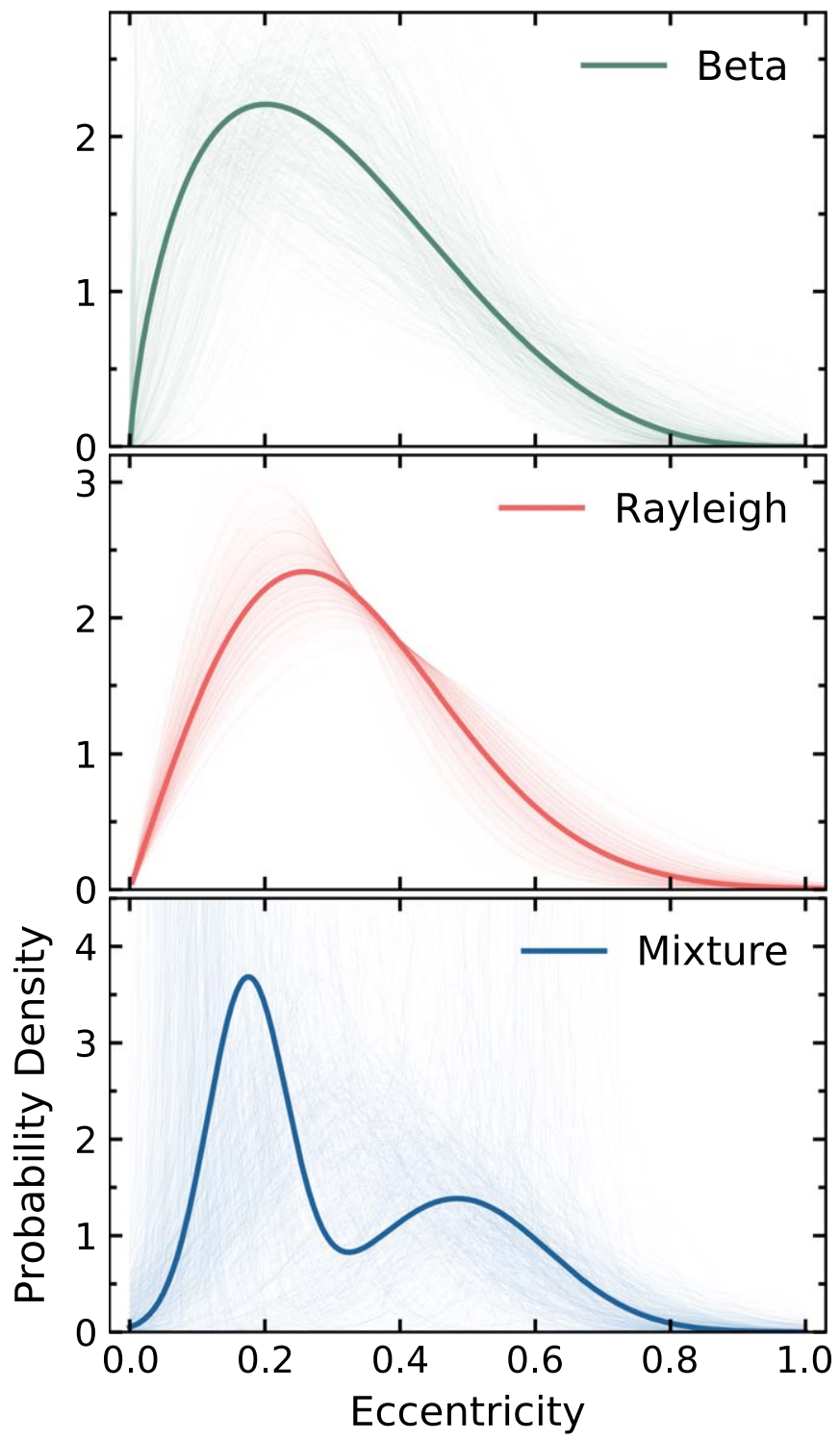

Figure 7. The eccentricity distributions of the catalog of 55 Warm Jupiter candidates discovered in Year 1 TESS FFIs (Table 4). The eccentricity distributions are inferred using hierarchical Bayesian modeling with the beta distribution (upper panel), the Rayleigh distribution (middle panel), and the two-component mixture distribution (lower panel) as the functional forms. Planets' eccentricities inferred from the beta distribution are generally lower than the eccentricities inferred from the Rayleigh distribution. The mixture model splits roughly $55 \%$ of the candidates into a low-e population centered at 0.16 , and the rest $45 \%$ into a high-e population centered at 0.49 .

for their sample of Kepler Hot Jupiters. The reason might be that the beta distribution has more flexible shapes compared to the Rayleigh distribution. For our sample of TESS Warm Jupiters, the eccentricity distribution spreads between 0 and 0.8 and peaks at $\sim 0.19$ for the beta distribution, whereas it peaks at $\sim 0.26$ for the Rayleigh distribution. For the two-component mixture model, the eccentricity distribution is well-constrained by a low- $e$ population centered at $\bar{e}=0.174_{-0.088}^{+0.114}$ and a high- $e$ population centered at $\bar{e}=0.484_{-0.095}^{+0.107}$. About $53 \pm 20 \%$ of the systems fall into the low-e population, and $47 \pm 20 \%$ of the systems fall into the high-e population. The corresponding widths of two distributions are $\sigma_{1}=0.059_{-0.043}^{+0.072}$ for the low- $e$ population and $\sigma_{2}=0.135_{-0.071}^{+0.063}$ for the high-e population. Naively speaking, the fraction of the systems categorized into the low-e population $(\sim 53 \%)$ could indicate the fraction of Warm Jupiters that originated from the disk migration and in situ formation; the fraction of the systems categorized into the high- $e$ population $(\sim 47 \%)$ could indicate the fraction of Warm Jupiters that originated from the high-eccentricity tidal migration. However, such a conclusion overlooks the evolution of Warm Jupiters' eccentricities, as discussed in Section 4. To correctly interpret the eccentricity distribution of a twocomponent mixture model, we will have to compare it with population predictions from different theorized origin channels of Warm Jupiters.

While we tested three functional forms for this distribution, we do not make any strong quantitative claims about the relative performance of each. Each model is qualitatively consistent with the others, within the uncertainties, and given the size of the data set and the dimensionality of the model, a formal model comparison (e.g., using the nested sampling algorithm employed by Kipping (2014b) to characterize the eccentricity distribution of radial velocity planets) would be computationally expensive and may not provide strong evidence for one model. We do find that the weights for each component of the mixture model are significantly inconsistent with zero, providing weak evidence that the two-component model is required to capture the distribution. Finally, as a robustness test for the eccentricity distribution, we tried randomly dropping $30 \%$ of the targets from our sample and conducting the same analysis on the new sample. We found the results to be consistent within $1 \sigma$ uncertainties.

\section{Discussion}

Here, we discuss our Warm-Jupiter-candidate catalog in the context of the TESS Extended Mission and ground-based follow-up observations. We also discuss the implication of our study on Warm Jupiter origins.

\subsection{TESS Extended Mission}

We identified 55 Warm Jupiters candidates in the Year 1 TESS FFIs. Many of these candidates will be revisited (at the time of this writing) during the TESS Extended Mission (e.g., in Year 3 data). The longer observing baseline will help with the TTV analysis and identify any additional planets in the system. About 20 out of the 55 candidates only have two transits observed during the TESS Prime Mission. The TESS Extended Mission will likely catch two more transits of these targets, to allow some preliminary TTV analysis. There is also a group of candidates with possible TTV signals (Figure 2). The extended mission data will allow us to examine the robustness of these signals. Besides that, the finer observing cadence of the extended mission data (i.e., from 30 minute to 10 minute) will improve the impact parameter characterization and the eccentricity analysis. In particular, the 19 possible Warm Jupiter candidates that are not selected in our catalog could be included if we can rule out the $b>1$ solution with the fine-cadence data. We also identify eight candidates with degenerate orbital periods listed in Table 5. The TESS extended mission data are likely to break the degeneracy of the orbital periods.

Most of our Warm Jupiter candidates have orbital periods of less than 20 days, due to their short observing baselines (i.e., 27 or 54 days). Combing the TESS Prime and Extended Mission data will allow the discovery of Warm Jupiters in the longer 
orbital period range, especially the targets identified as singletransit events in the Year 1 data.

\subsection{Ground-based Follow-up Observations}

Ground-based follow-up observations are essential to validate planet candidates in our catalog. Due to the coarse angular resolution of TESS, ground-based photometric followup will be required to confirm on-target transits. If the target has no aperture contamination, radial velocity follow-up will further rule out brown dwarfs or low-mass stars. As discussed in Section 2.3, the TESS community has already made great progress in validating planet candidates and ruling out FPs in our catalog. Mass measurements will be essential to examine the giant planet mass-metallicity relation (Thorngren et al. 2016) and connection to the host star metallicity (e.g., Teske et al. 2019) for Warm Jupiters.

Here, we propose a follow-up strategy for the Warm Jupiter candidates. First, we propose to prioritize follow-up of candidates showing evidence of high eccentricities. The high- $e$ planets play an important role in constructing the eccentricity distribution. Validation will ensure that their contribution to the populationwide eccentricity distribution is real-and moreover, confirm or rule out the high-eccentricity tidal migration scenario as one of the predominant origin channels contributing to the Warm Jupiter population. Radial velocity follow-up will further break the degeneracy between eccentricity and argument of periapse. A list of high-e candidates that have not yet been confirmed (130415266, 147660886, 24358417, 290403522, 395113305, 180989820, 464300749, 343936388) can be found in Section 4 and Table 4. Second, we propose to prioritize candidates showing possible TTV signals. The large transits depths of our candidates (i.e., several parts per thousand) make them feasible for groundbased photometric follow-up observations. For example, in the case of TRAPPIST telescopes, transit events with depths greater than 2.5 parts per thousand will readily be detected. In general, candidates with $>2 \sigma$ TTV detections are worthwhile to follow up (see Figure 2). We also listed four targets (150299840, 382200986, 371188886, 343936388) showing possible sinusoidal TTV signals in Section 4. Extensive transit follow-up will help to detect any non-transiting, nearby planets and shed light on the dynamical history of the system. Finally, we recommend prioritizing the remaining targets given their transmission spectroscopy metric (TSM; Kempton et al. 2018) for follow-up atmospheric characterization observations. In Table 4, Column 13, we calculate a TSM score using the empirical mass-radius relationship of Chen \& Kipping (2017) for each target on a scale of 1-100, with 100 being most favorable. The confirmation of these targets will help to select ideal candidates for Warm Jupiter atmospheric characterization for future missions (e.g., JWST).

Follow-up observations on candidates with missing information listed in Table 5 are also important. Photometric and radial velocity follow-up observations will help to break the orbital period degeneracy due to TESS observation gaps. Furthermore, characterization of stellar densities will allow photoeccentric analysis.

\subsection{Implication of the Eccentricity Distribution}

We identified a large sample of Warm Jupiter candidates to conduct a preliminary eccentricity distribution study of the population using the "photoeccentric" approach to constrain the eccentricity from the transit light curve. There are several caveats with regard to our results. Although we have incorporated uncertainties in stellar density, systematic errors in stellar parameters could impact our inference of eccentricities. Since many of the candidates have not yet been statistically validated, our interpretation of the eccentricity distribution could also be compromised by astrophysical false positives, e.g., binaries and brown dwarfs, which may have different dynamical histories and thus different eccentricity distributions from Warm Jupiters'. Due to the short TESSsector observing baseline, most of our candidates have orbital periods less than 20 days. The eccentricity distributions of longer-period Warm Jupiters are not addressed in detail in this work.

In our preliminary study, we found both single-component models (i.e., the beta distribution and the Rayleigh distribution) and a two-component model could be used as the functional forms of the eccentricity distribution. Our two-component model is flexible enough to describe both single-component and two-component distributions, benefiting from its five free parameters. The two-component eccentricity distribution showed that slightly more than half of the Warm Jupiters have nearly circular orbits, in support of the disk migration and in situ origin scenarios; slightly less than half of the Warm Jupiters have moderately to highly elliptical orbits, in support of high-eccentricity tidal migration. However, as discussed in Section 5, evolution of planetary eccentricities could modify the shape of the distribution. A statistical study on a clean Warm Jupiter catalog will require extensive ground-based follow-up observations and is deferred to future work. In future studies, the eccentricity distribution can be compared more directly with predictions from different origin theories (e.g., Anderson et al. 2020) to shed light on one or more origin channels that predominantly contribute to the Warm Jupiter population.

Benefiting from the extensive follow-up observations, a dozen of the Warm Jupiter candidates in the catalog have been confirmed. In Appendix $\mathrm{C}$, we demonstrate one approach to incorporate eccentricity measurements from different sources However, as will be discussed in Appendix C, further experiments would be necessary to robustly account for variations in selection effects.

We note that the eccentricity distributions we found here are the observed eccentricity distributions of a sample of transiting Warm Jupiter candidates. While we have taken account of the transit probability, to correct the observation biases for the eccentricity inferences, the detection efficiency of the transit search will also be a function of the eccentricity (e.g., targets with high eccentricities will have a better chance to transit; Kipping 2014b). To find the intrinsic eccentricity distribution, we will need to weight planets differently in the hierarchical Bayesian model according to their detection efficiency, which will depend on its $\rho_{\star}, e, \omega$, and $P$. To more directly compare a model to the observations, in future work we can forward model the detection efficiency to characterize the eccentricity distribution of simulated detected transiting planets. The vetting efficiency is less of a concern since the large transit $\mathrm{S} / \mathrm{Ns}$ of giant planets make them readily vetted, and they are readily detected around our bright targets even if a high eccentricity shortens their transit duration.

It will also be interesting to see how the eccentricity distribution varies as a function of semimajor axis. In a preliminary study, we separated our candidates into two groups, 
$a<0.1$ au (29 candidates) and $a>0.1$ au (26 candidates), and inferred their eccentricity distributions. We found planets with greater semimajor axes have higher median eccentricities compared to those of planets with smaller semimajor axes. This finding is consistent with theories, since both the in situ formation scenario that leads to planet-planet scattering and the high-e migration scenario predict an increase of eccentricity as the semimajor axis increases. However, the tidal circularization effect also needs to be taken into account here because, with $a<0.1 \mathrm{au}$, some planets could have been circularized in their systems' lifetime. The issue can be solved by increasing the number of semimajor axis bins if more Warm Jupiters at large semimajor axes are detected.

\section{Summary}

We systematically searched for Warm Jupiter candidates, i.e., planets greater than 6 Earth radii with orbital periods of 8-200 days, around host stars brighter than Tmag of 12 in Year 1 TESS Full-Frame Images (Figure 1). We characterized each candidate's TESS light curve with a quadratic limb darkening transit model along with GPs. For candidates with more than two transits, we analyzed their TTVs (Figure 2). We inferred each planet's eccentricity using the "photoeccentric" effect (Figure 3 and 4). In Table 4, we tabulate the catalog of Warm Jupiter candidates with their host star and planet properties. Furthermore, we derived the preliminary eccentricity distribution of the Warm-Jupiter-candidate catalog using HBM (Figure 6). We investigated three functional forms for the eccentricity distribution - the beta distribution, the Rayleigh distribution, and the mixture distribution - and found a set of well-constrained hyperparameters for each functional form (Figure 7 and Table 6). Extensive ground-based follow-up observations will be required to identify FPs in the sample and to construct a clean Warm Jupiter catalog. We proposed a follow-up strategy in Section 6.2. In future studies, the eccentricity distribution can be directly compared with predictions from different origin theories, with detection effects accounted for, to shed light on origin channels that predominantly contribute to the Warm Jupiter population.

This research made use of exoplanet (Foreman-Mackey et al. 2019) and its dependencies (Kipping 2013; RAstropy Collaboration et al. 2013; Theano Development Team 2016; Salvatier et al. 2016; Astropy Collaboration et al. 2018; Foreman-Mackey 2018; Foreman-Mackey et al. 2019, 2017; Luger et al. 2019; Agol et al. 2020). This research made use of Lightkurve, a Python package for Kepler and TESS data analysis (Lightkurve Collaboration et al. 2018). This research made use of astroquery (Ginsburg et al. 2019). Computations for this research were performed on the Pennsylvania State University's Institute for CyberScience Advanced CyberInfrastructure (ICS-ACI). This content is solely the responsibility of the authors and does not necessarily represent the views of the Institute for CyberScience.

This work has made use of data from the European Space Agency (ESA) mission Gaia (https://www.cosmos.esa.int/ gaia), processed by the Gaia Data Processing and Analysis Consortium (DPAC, https://www.cosmos.esa.int/web/gaia/ dpac/consortium). Funding for the DPAC has been provided by national institutions, in particular the institutions participating in the Gaia Multilateral Agreement.
We acknowledge the use of TESS High Level Science Products (HLSP) produced by the Quick-Look Pipeline (QLP) at the TESS Science Office at MIT, which are publicly available from the Mikulski Archive for Space Telescopes (MAST). Funding for the TESS mission is provided by NASA's Science Mission directorate.

This paper includes data collected by the TESS mission, which are publicly available from the Mikulski Archive for Space Telescopes (MAST). Resources supporting this work were provided by the NASA High-End Computing (HEC) Program through the NASA Advanced Supercomputing (NAS) Division at Ames Research Center for the production of the SPOC data products.

This research has made use of the NASA Exoplanet Archive, which is operated by the California Institute of Technology, under contract with the National Aeronautics and Space Administration under the Exoplanet Exploration Program.

This work makes use of observations from the LCOGT network (proposal IDs: 2020B-0189, 2020B-0202).

The research leading to these results has received funding from the ARC grant for Concerted Research Actions, financed by the Wallonia-Brussels Federation. TRAPPIST is funded by the Belgian Fund for Scientific Research (Fond National de la Recherche Scientifique, FNRS) under the grant FRFC 2.5.594.09.F, with the participation of the Swiss National Science Foundation (SNF). TRAPPIST-North is a project funded by the University of Liege (Belgium), in collaboration with Cadi Ayyad University of Marrakech (Morocco) MG and EJ are F.R.S.-FNRS Senior Research Associate. This publication benefits from the support of the French Community of Belgium in the context of the FRIA Doctoral Grant awarded to MT.

This research received funding from the European Research Council (ERC) under the European Union's Horizon 2020 research and innovation program (grant agreement No. 803193/ BEBOP), and from the Science and Technology Facilities Council (STFC; grant No. ST/S00193X/1).

R.B. acknowledges support from FONDECYT Project 11200751, CORFO project No. 14ENI2-26865.

R.B. and A.J. acknowledge support from ANID-Millennium Science Initiative-ICN12_009. A.J. acknowledges additional support from FONDECYT project 1210718.

This work has been carried out within the framework of the NCCR PlanetS supported by the Swiss National Science Foundation. We made use of PlanetS' Data \& Analysis Center for Exoplanets (DACE), which is available at https://dace. unige.ch.

This research has made use of the Exoplanet Follow-up Observation Program website, which is operated by the California Institute of Technology, under contract with the National Aeronautics and Space Administration under the Exoplanet Exploration Program.

We gratefully acknowledge support by NASA XRP NNX16AB50G, NASA TESS GO 80NSSC18K1695, and the Alfred P. Sloan Foundation's Sloan Research Fellowship. The Center for Exoplanets and Habitable Worlds is supported by the Pennsylvania State University, the Eberly College of Science, and the Pennsylvania Space Grant Consortium. J.D. gratefully acknowledges support and hospitality from the predoctoral program at the Center for Computational Astrophysics, Flatiron Institute. Research at the Flatiron Institute is supported by the Simons Foundation. J.D. thanks the Flatiron 
Astronomical Data Group and Planet Formation Group for helpful discussions. J.D. gratefully acknowledges Angie Wolfgang and Tom Loredo for inspiring lectures on Bayesian statistics. J.D. thanks Eric Ford, Kassandra Anderson, Juliette Becker, Vincent Van Eylen, Matthias He, Sarah Millholland, Ruth Murray-Clay, Cristobal Petrovich, and Keivan Stassun for helpful discussions.

Facilities: TESS, Gaia, LCOGT, Exoplanet Archive. Software: Arviz (Kumar et al. 2019), AstroImageJ (Collins et al. 2017), astropy (RAstropy Collaboration et al. 2013; Astropy Collaboration et al. 2018), astroquery (Ginsburg et al. 2019), celerite (Foreman-Mackey et al. 2017; ForemanMackey 2018), exoplanet (Foreman-Mackey et al. 2019), Jupyter (Kluyver et al. 2016), lightkurve (Lightkurve Collaboration et al. 2018), Matplotlib (Hunter 2007;
Droettboom et al. 2016), NumPy (van der Walt et al. 2011; Harris et al. 2020), pandas (Wes McKinney 2010), PyMC3 (Salvatier et al. 2016), SciPy (Virtanen et al. 2020), starry (Luger et al. 2019), TAPIR (Jensen 2013), Theano (Theano Development Team 2016).

\section{Appendix A Light Curves of non-TOI Candidates}

We present the FFI light curves of non-TOI candidates obtained from the QLP. In each figure, we plot the target's full light curve in gray dots, the trimmed light curves in black dots, and the best-fitted light curve models in blue lines. See Figure A1 for demonstration.

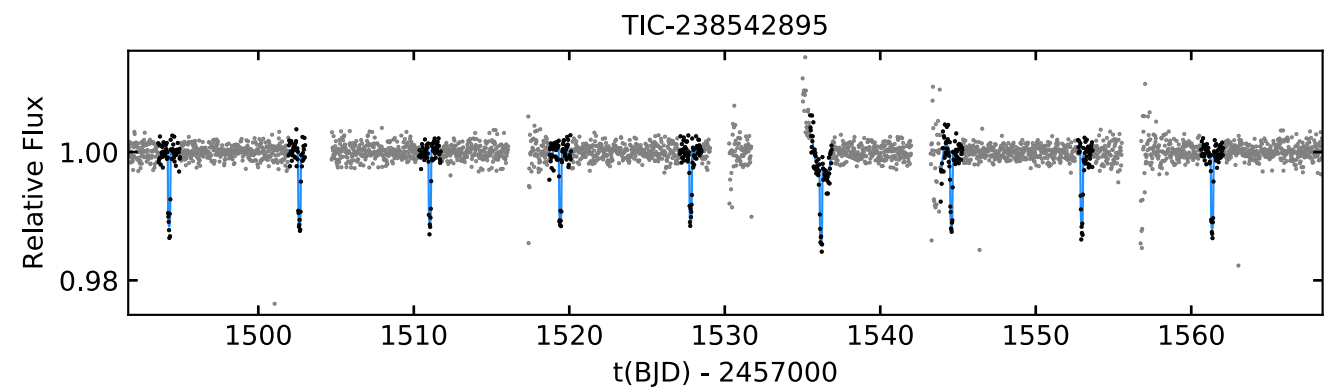

Figure A1. TESS Full-Frame-Image light curves of TIC-238542895 processed by the Quick Look Pipeline. A full light curve is plotted as gray dots, the trimmed light curves used for modeling are colored in black, and the best-fitted light curve models are shown as blue lines. The complete figure set (19 figures) is available in the online journal.

(The complete figure set (19 images) is available.) 


\section{Appendix B}

\section{Hyperparameter Posterior Distributions}

Corner plots for the posterior distributions of the hyperparameters assuming the beta distribution as the functional form of the eccentricity distribution (Figure B1) and the two-component mixture distribution as the functional form (Figure B2). See Section 5 for more details.

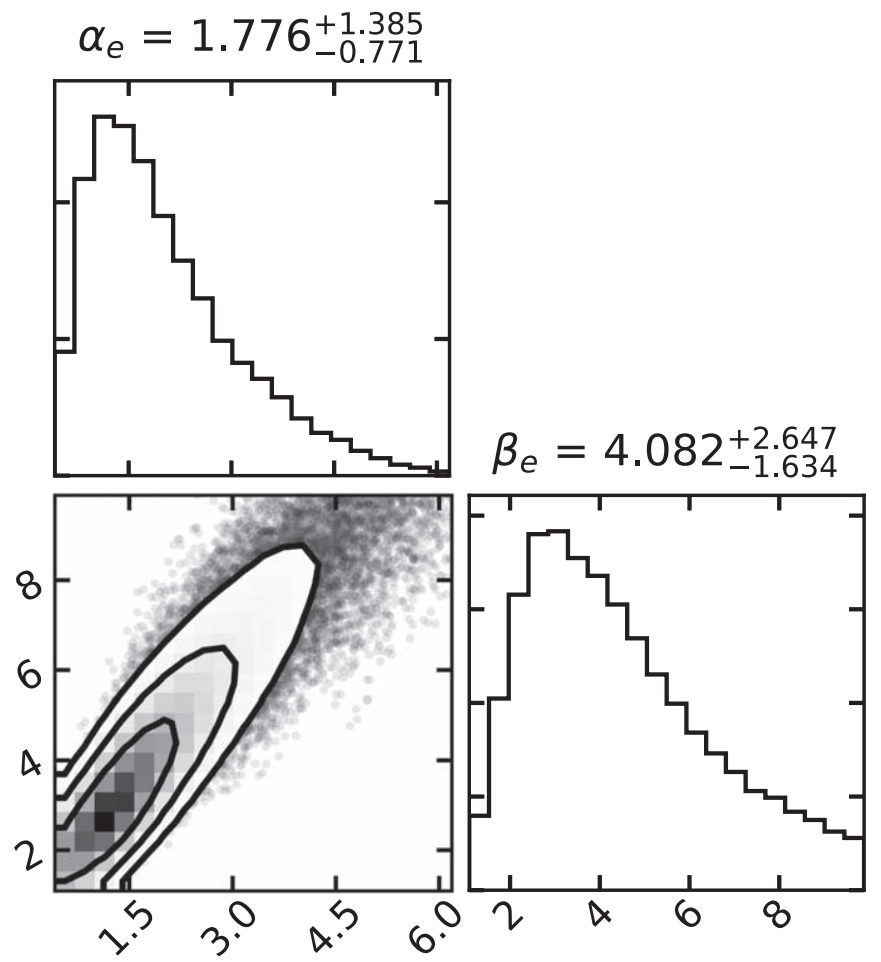

Figure B1. Posterior distributions of hyperparameters using the beta distribution as the functional form of the eccentricity distribution. The probability distribution follows $p\left(e \mid \alpha_{e}, \beta_{e}\right)=e^{\alpha-1}(1-e)^{\beta-1} / B(\alpha, \beta)$, where $B(\alpha, \beta)=\Gamma(\alpha) \Gamma(\beta) / \Gamma(\alpha+\beta)$ and $\Gamma$ is the gamma function. 


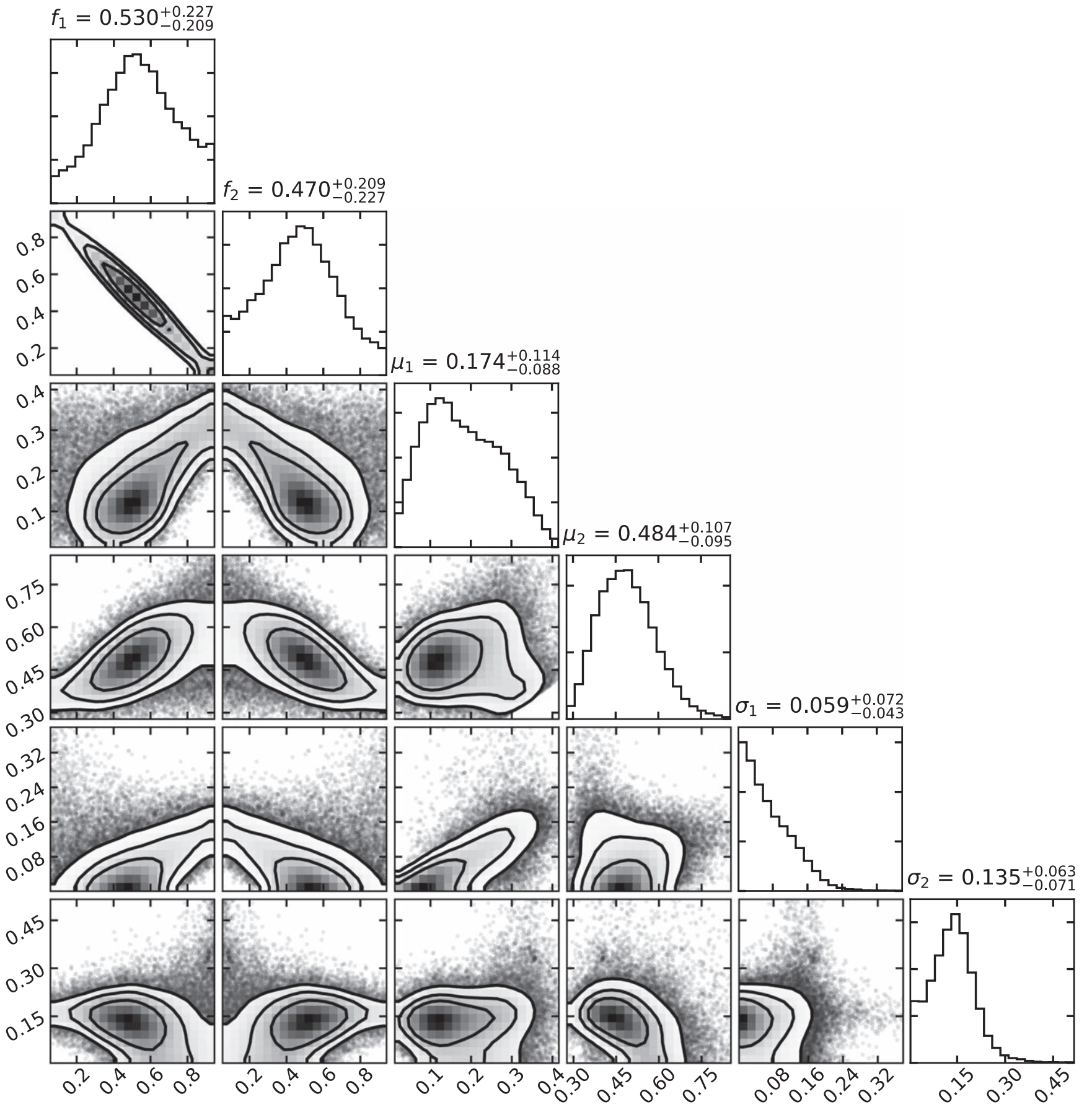

Figure B2. Posterior distributions of hyperparameters using the two-component mixture distribution as the functional form of the eccentricity distribution. The probability distribution follows $p\left(e \mid f_{1}, f_{2}, \mu_{1}, \mu_{2}, \sigma_{1}, \sigma_{2}\right)=f_{1} \mathcal{N}\left(e \mid \mu_{1}, \sigma_{1}^{2}\right)+f_{2} \mathcal{N}\left(e \mid \mu_{2}, \sigma_{2}^{2}\right)$, where $\mathcal{N}$ is the normal distribution.

\section{Appendix C}

Incorporating Eccentricity Measurements from Different Sources

Benefiting from the extensive follow-up observations of TESS planet candidates, 12 targets in our catalog have been confirmed. These confirmed planets have radial-velocity or TTV-constrained eccentricities that can be used for the eccentricity distribution inference. Here, we conduct the HBM using a framework similar to the one shown in Section 5, but include the information of planets with better-constrained eccentricities. In Figure $\mathrm{C} 1$, we present the graphic model of the extended hierarchical Bayesian model. Planets are separated into two panels: the left panel is for the ones without further constrained eccentricities, so we continue to adopt the photoeccentric approach (similar to Figure 6); the right panel is for the ones with further constrained eccentricities from ground-based follow-up observations. The median and $1 \sigma$ uncertainty of eccentricities are extracted from literature (see Table 4 column 17 for references) to construct the observed parameters $\hat{e}_{j}$ and $\sigma_{\hat{e}_{i}}$. Planets that have been followed up have much smaller eccentricity uncertainties $\left(\sigma_{e} \sim 0.01\right)$ compared to the ones that have only been analyzed from their light curves $\left(\sigma_{e} \sim 0.2\right)$. The inclusion of these precise eccentricity measurements risks biasing the inferred eccentricity distribution, since they are likely to be drawn from a 


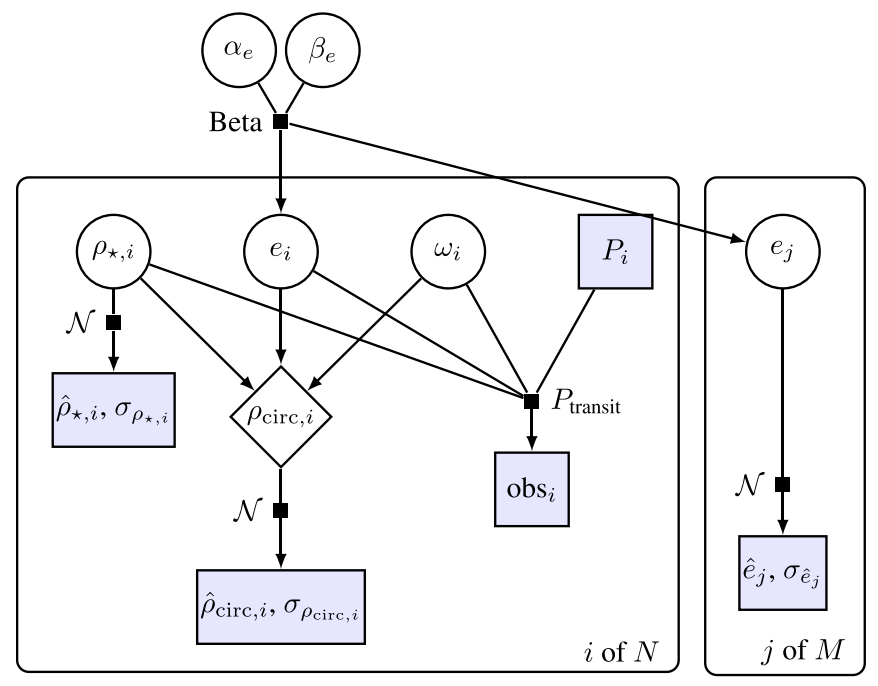

Figure C1. Graphic model of the extended hierarchical Bayesian model. This model is an extension of the model shown in Figure 6. Since a few planets in our catalog have further constrained eccentricities from ground-based followup observations, we adopt their eccentricity posteriors to evaluate the eccentricity distribution directly. For planets without further constrained eccentricities, we continue to adopt the photoeccentric approach.

Table C1

Summary of the Posteriors of the Hyperparameters for the Extended Hierarchical Bayesian Models

\begin{tabular}{lcc}
\hline \hline Distribution & \multicolumn{2}{c}{ Hyperparameter Posteriors } \\
\hline Beta & $\alpha_{e}$ & $\beta_{e}$ \\
& $1.053_{-0.285}^{+0.379}$ & $2.771_{-0.778}^{+1.036}$ \\
\hline Rayleigh & $\sigma_{e}$ & \\
& $0.243_{-0.025}^{+0.028}$ & $68 \%$ HPD intervals \\
\hline Mixture & Mode & {$[0.742,1.000],[0.078,0.411]$} \\
$f_{1}$ & $0.947,0.228$ & {$[0.150,0.330],[0.013,0.019]$} \\
$\mu_{1}$ & $0.271,0.016$ & {$[0.074,0.193],[0.009,0.013]$} \\
$\sigma_{1}$ & $0.148,0.011$ & {$[0.000,0.258],[0.589,0.922]$} \\
$f_{2}$ & $0.053,0.772$ & {$[0.283,0.478]$} \\
$\mu_{2}$ & 0.350 & {$[0.091,0.224]$} \\
$\sigma_{2}$ & 0.159 & \\
\hline
\end{tabular}

Notes. We report the medians and $68 \%$ credible intervals of the posteriors for the beta and Rayleigh distributions. For the mixture model, the posterior distributions are bimodal, as shown in Figure C3. We instead report the modes and the $68 \%$ highest posterior density intervals.

different underlying population than the full sample, and this subset will be censored by different selection effects. Furthermore, there exist more recently discovered likely high-e planets that have yet to be followed up. This model is a demonstration of a method for incorporating eccentricity measurements from different sources, but further experiments would be necessary to robustly account for variations in selection effects.

In Figure C2, we show the eccentricity distributions using three different functional forms inferred from the extended model. Inferred hyperparameters can be found in Table $\mathrm{Cl}$. Comparing to the eccentricity distributions inferred without including these information (i.e., Figure 7), the Rayleigh distribution is consistent with the previous distribution within $1 \sigma$ uncertainty of the hyperparameter. For the beta distribution, however, it is more right-skewed and has a mode eccentricity close to zero. For the

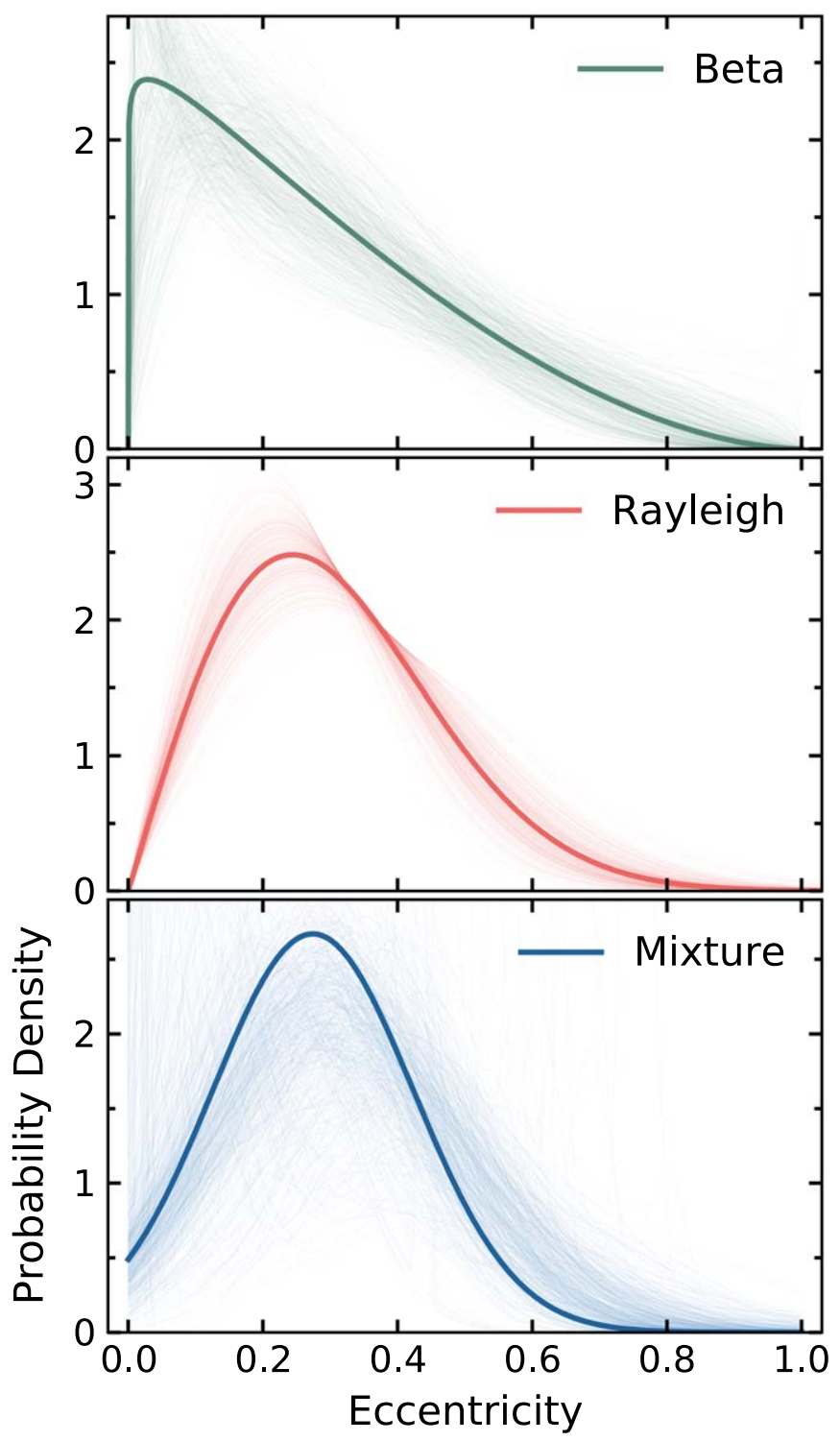

Figure C2. The eccentricity distributions inferred from the extended hierarchical Bayesian model shown in Figure $\mathrm{C} 1$. The extended model includes the information of confirmed planets with further constrained eccentricities. Comparing to the eccentricity distributions inferred without including these information (i.e., Figure 7), the Rayleigh distribution is consistent with the previous distribution (i.e., the hyperparameter is consistent within $1 \sigma$ ), whereas both the beta distribution and the mixture distribution present obvious changes in the distributions. The beta distribution becomes more right-skewed. The mixture distribution is composed of a single component, instead of two, and now has a shape similar to that of the Rayleigh distribution.

mixture model, a single-component distribution is favored over the two-component distribution. As shown in Figure C3, the joint and marginal posterior distributions of the hyperparameters are bimodal but have a strong preference for a single-component model. The best-fitted mixture distribution now has a shape similar to the Rayleigh distribution. The changes on the eccentricity distributions are likely caused by the small eccentricity uncertainties of the confirmed planets, as discussed earlier. To examine the statement, we modify the eccentricity uncertainties of the confirmed planets to the typical eccentricity uncertainties from the photoeccentric analysis (i.e., $\sigma_{e} \sim 0.2$ ) and redo the analysis. We find the inferred eccentricity distributions are consistent with the ones shown in Figure 7. 


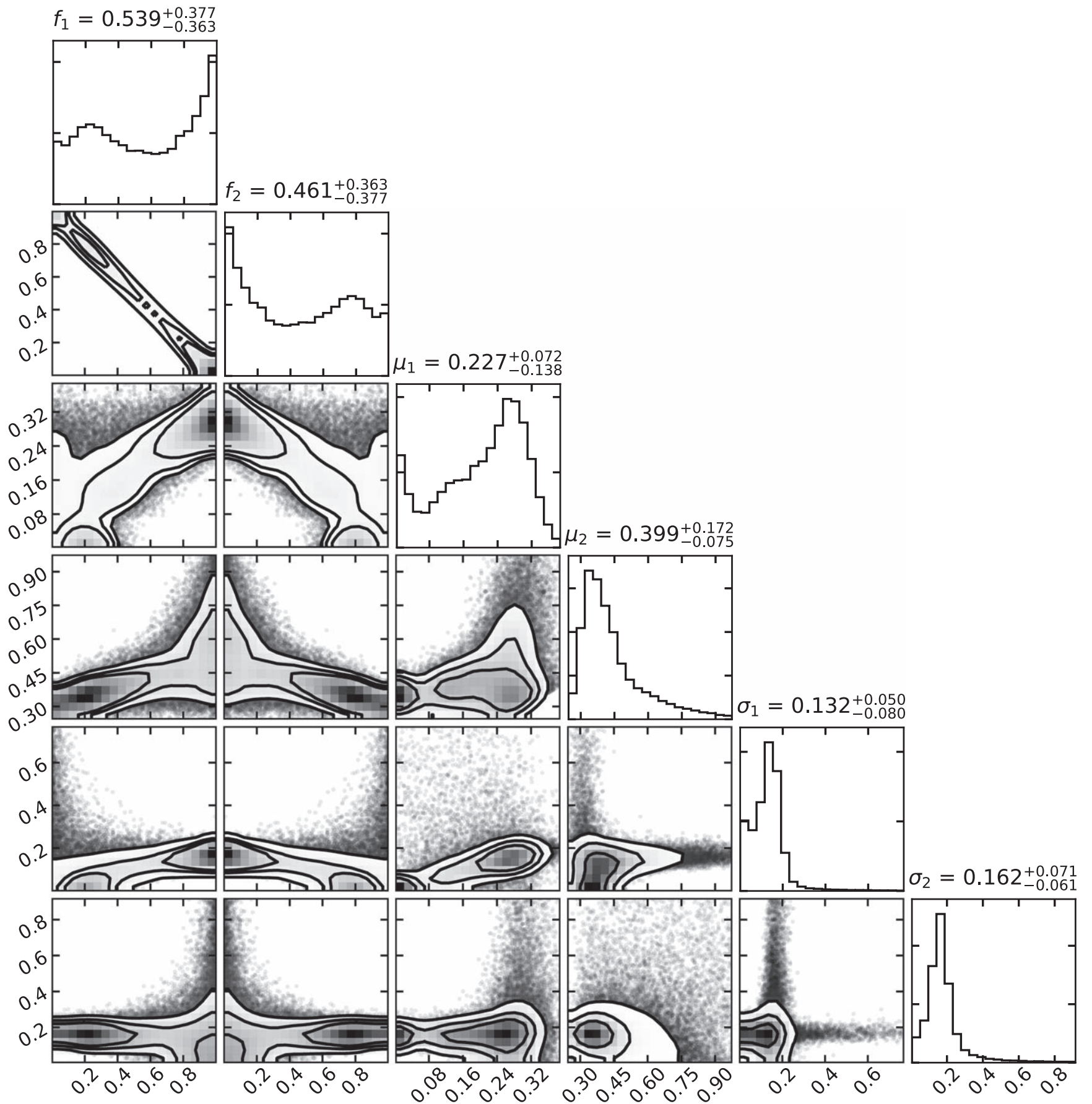

Figure C3. Posterior distributions of hyperparameters using the mixture distribution as the functional form of the eccentricity distribution for the extended hierarchical Bayesian model. The distributions are bimodal, with a preference for a single-component normal distribution.

\section{ORCID iDs}

Jiayin Dong (i) https://orcid.org/0000-0002-3610-6953 Chelsea X. Huang (i) https://orcid.org/0000-0003-0918-7484

Rebekah I. Dawson (ํ) https://orcid.org/0000-0001-9677-1296 Daniel Foreman-Mackey (i) https://orcid.org/0000-00029328-5652

Karen A. Collins ำ https://orcid.org/0000-0001-6588-9574 Samuel N. Quinn (1) https://orcid.org/0000-0002-8964-8377 Jack J. Lissauer (ib https://orcid.org/0000-0001-6513-1659 Thomas Beatty i https://orcid.org/0000-0002-9539-4203 Billy Quarles (i) https://orcid.org/0000-0002-9644-8330 Lizhou Sha $(1)$ https://orcid.org/0000-0001-5401-8079
Avi Shporer (i) https://orcid.org/0000-0002-1836-3120 Stephen R. Kane (1) https://orcid.org/0000-0002-7084-0529 Khalid Barkaoui (10 https://orcid.org/0000-0003-1464-9276 Zouhair Benkhaldoun (10 https://orcid.org/0000-0001-6285-9847 Rafael Brahm (1) https://orcid.org/0000-0002-9158-7315 François Bouchy (i) https://orcid.org/0000-0002-7613-393X Theron W. Carmichael (1) https://orcid.org/0000-00016416-1274

Kevin I. Collins (1) https://orcid.org/0000-0003-2781-3207 Dennis M. Conti (1) https://orcid.org/0000-0003-2239-0567 Nicolas Crouzet (ㄷ) https://orcid.org/0000-0001-7866-8738 Georgina Dransfield (10) https://orcid.org/0000-00023937-630X 
Phil Evans (1) https://orcid.org/0000-0002-5674-2404

Tianjun Gan (1) https://orcid.org/0000-0002-4503-9705

Michaël Gillon (1) https://orcid.org/0000-0003-1462-7739

Nolan Grieves 우 https://orcid.org/0000-0001-8105-0373

Tristan Guillot (1) https://orcid.org/0000-0002-7188-8428

Coel Hellier (10) https://orcid.org/0000-0002-3439-1439

Emmanuël Jehin (ㄱ) https://orcid.org/0000-0001-8923-488X

Eric L. N. Jensen (1) https://orcid.org/0000-0002-4625-7333

Andres Jordán (i) https://orcid.org/0000-0002-5389-3944

John F. Kielkopf (i) https://orcid.org/0000-0003-0497-2651

Djamel Mékarnia (i) https://orcid.org/0000-0001-5000-7292

Louise D. Nielsen (1) https://orcid.org/0000-0002-5254-2499

Francisco J. Pozuelos (i) https://orcid.org/0000-0003-

1572-7707

François-Xavier Schmider (i) https://orcid.org/0000-00033914-3546

Richard P. Schwarz (10 https://orcid.org/0000-0001-8227-1020

Chris Stockdale (1) https://orcid.org/0000-0003-2163-1437

Thiam-Guan Tan (1) https://orcid.org/0000-0001-5603-6895

Amaury H. M. J. Triaud (ํ) https://orcid.org/0000-0002-

5510-8751

Gavin Wang (1) https://orcid.org/0000-0003-3092-4418

George Ricker 나 https://orcid.org/0000-0003-2058-6662

Roland Vanderspek (i) https://orcid.org/0000-0001-6763-6562

David W. Latham (1) https://orcid.org/0000-0001-9911-7388

Sara Seager (10) https://orcid.org/0000-0002-6892-6948

Joshua N. Winn (1) https://orcid.org/0000-0002-4265-047X

Jon M. Jenkins (i) https://orcid.org/0000-0002-4715-9460

Ismael Mireles (다 https://orcid.org/0000-0002-4510-2268

Daniel A. Yahalomi (1) https://orcid.org/0000-0003-

4755-584X

Edward H. Morgan (1) https://orcid.org/0000-0003-1447-6344

Elisa V. Quintana (1) https://orcid.org/0000-0003-1309-2904

Mark E. Rose (1i) https://orcid.org/0000-0003-4724-745X

Jeffrey C. Smith (1) https://orcid.org/0000-0002-6148-7903

\section{References}

Addison, B. C., Wright, D. J., Nicholson, B. A., et al. 2021, MNRAS, 502,3704

Agol, E., Luger, R., \& Foreman-Mackey, D. 2020, AJ, 159, 123

Anderson, K. R., \& Lai, D. 2017, MNRAS, 472, 3692

Anderson, K. R., Lai, D., \& Pu, B. 2020, MNRAS, 491, 1369

Arras, P., \& Socrates, A. 2010, ApJ, 714, 1

Astropy Collaboration, Robitaille, T. P., Tollerud, E. J., et al. 2013, A\&A, 558, A33

Astropy Collaboration, Price-Whelan, A. M., Sipőcz, B. M., et al. 2018, AJ, 156, 123

Baruteau, C., Crida, A., Paardekooper, S. J., et al. 2014, in Protostars and Planets VI, ed. H. Beuther et al. (Tucson, AZ: Univ. Arizona Press), 667

Batygin, K., Bodenheimer, P. H., \& Laughlin, G. P. 2016, ApJ, 829, 114 Betancourt, M. 2017, arXiv:1701.02434

Bodenheimer, P., Lin, D. N. C., \& Mardling, R. A. 2001, ApJ, 548, 466 Boley, A. C., Granados Contreras, A. P., \& Gladman, B. 2016, ApJL, 817, L17

Bouma, L. G., Hartman, J. D., Brahm, R., et al. 2020, AJ, 160, 239

Brahm, R., Espinoza, N., Jordán, A., et al. 2019, AJ, 158, 45

Brahm, R., Nielsen, L. D., Wittenmyer, R. A., et al. 2020, AJ, 160, 235

Burke, C. J. 2008, ApJ, 679, 1566

Carmichael, T. W., Quinn, S. N., Mustill, A. J., et al. 2020, AJ, 160, 53

Carmichael, T. W., Quinn, S. N., Zhou, G., et al. 2021, AJ, 161, 97

Chen, J., \& Kipping, D. 2017, ApJ, 834, 17

Collins, K. A., Collins, K. I., Pepper, J., et al. 2018, AJ, 156, 234

Collins, K. A., Kielkopf, J. F., Stassun, K. G., \& Hessman, F. V. 2017, AJ, 153,77

Dawson, R. I., Huang, C. X., Brahm, R., et al. 2021, AJ, 161, 161

Dawson, R. I., Huang, C. X., Lissauer, J. J., et al. 2019, AJ, 158, 65

Dawson, R. I., \& Johnson, J. A. 2012, ApJ, 756, 122
Dawson, R. I., \& Johnson, J. A., 2018, ARA\&A, 56, 175

Dawson, R. I., \& Murray-Clay, R. A. 2013, ApJL, 767, L24

Dawson, R. I., Murray-Clay, R. A., \& Johnson, J. A. 2015, ApJ, 798, 66

Debras, F., Baruteau, C., \& Donati, J.-F. 2021, MNRAS, 500, 1621

Dong, S., Katz, B., \& Socrates, A. 2014, ApJL, 781, L5

Dotter, A., Chaboyer, B., Jevremović, D., et al. 2008, ApJS, 178, 89

Droettboom, M., Hunter, J., Caswell, T. A., et al. 2016, Matplotlib: Matplotlib v1.5.1, Zenodo doi:10.5281/zenodo.44579

Duffell, P. C., \& Chiang, E. 2015, ApJ, 812, 94

Eggleton, P. P., Kiseleva, L. G., \& Hut, P. 1998, ApJ, 499, 853

Fabrycky, D. C., Lissauer, J. J., Ragozzine, D., et al. 2014, ApJ, 790, 146

Ford, E. B., \& Rasio, F. A. 2008, ApJ, 686, 621

Foreman-Mackey, D. 2016, JOSS, 1, 24

Foreman-Mackey, D. 2018, RNAAS, 2, 31

Foreman-Mackey, D., Agol, E., Ambikasaran, S., \& Angus, R. 2017, AJ, 154,220

Foreman-Mackey, D., Czekala, I., Luger, R., et al. 2019, dfm/exoplanet: exoplanet v0.2.1, Zenodo doi:10.5281/zenodo.3462740

Frelikh, R., Jang, H., Murray-Clay, R. A., \& Petrovich, C. 2019, ApJL, 884, L47

Gaia Collaboration, Prusti, T., de Bruijne, J. H. J., et al. 2016, A\&A, 595, A1 Gaia Collaboration, Brown, A. G. A., Vallenari, A., et al. 2018, A\&A, 616, A1 Ginsburg, A., Sipőcz, B. M., Brasseur, C. E., et al. 2019, AJ, 157, 98 Goldreich, P., \& Tremaine, S. 1980, ApJ, 241, 425

Guerrero, N. M., Seager, S., Huang, C. X., et al. 2021, ApJS, 254, 39

Guillot, T., \& Showman, A. P. 2002, A\&A, 385, 156

Harris, C. R., Jarrod Millman, K., van der Walt, S. J., et al. 2020, Natur, 585,357

Hartman, J. D., \& Bakos, G. Á. 2016, A\&C, 17, 1

He, M. Y., Ford, E. B., \& Ragozzine, D. 2019, MNRAS, 490, 4575

Hellier, C., Anderson, D. R., Bouchy, F., et al. 2019, MNRAS, 482, 1379

Hellier, C., Anderson, D. R., Collier Cameron, A., et al. 2017, MNRAS, 465,3693

Hobson, M. J., Brahm, R., Jordán, A., et al. 2021, AJ, 161, 235

Hoffman, M. D., \& Gelman, A. 2011, arXiv:1111.4246

Huang, C., Wu, Y., \& Triaud, A. H. M. J. 2016, ApJ, 825, 98

Huang, C. X., Quinn, S. N., Vanderburg, A., et al. 2020a, ApJL, 892, L7

Huang, C. X., Vanderburg, A., Pál, A., et al. 2020b, RNAAS, 4, 204

Huang, C. X., Vanderburg, A., Pál, A., et al. 2020c, RNAAS, 4, 206

Huang, X., Burt, J., Vanderburg, A., et al. 2019, AAS Meeting Abstracts, 233, 209.08

Hunter, J. D. 2007, CSE, 9, 90

Ida, S., \& Lin, D. N. C. 2008, ApJ, 673, 487

Ida, S., \& Makino, J. 1992, Icar, 96, 107

Jackson, J. M., Dawson, R. I., \& Zalesky, J. 2019, AJ, 157, 166

Jehin, E., Gillon, M., Queloz, D., et al. 2011, Msngr, 145, 2

Jenkins, J. M., Caldwell, D. A., \& Borucki, W. J. 2002, ApJ, 564, 495

Jenkins, J. M., Caldwell, D. A., Chandrasekaran, H., et al. 2010, ApJL, 713, L87

Jensen, E. 2013, Tapir: A web interface for transit/eclipse observability, Astrophysics Source Code Library. ascl:1306.007

Jones, H. R. A., Butler, R. P., Tinney, C. G., et al. 2003, MNRAS, 341, 948

Jordán, A., Brahm, R., Espinoza, N., et al. 2020, AJ, 159, 145

Kane, S. R., Ciardi, D. R., Gelino, D. M., \& von Braun, K. 2012, MNRAS, 425,757

Kane, S. R., \& Raymond, S. N. 2014, ApJ, 784, 104

Kempton, E. M. R., Bean, J. L., Louie, D. R., et al. 2018, PASP, 130, 114401

Kipping, D., Nesvorný, D., Hartman, J., et al. 2019, MNRAS, 486, 4980

Kipping, D. M. 2013, MNRAS, 435, 2152

Kipping, D. M. 2014a, MNRAS, 440, 2164

Kipping, D. M. 2014b, MNRAS, 444, 2263

Kipping, D. M., Dunn, W. R., Jasinski, J. M., \& Manthri, V. P. 2012, MNRAS, 421,1166

Kluyver, T., Ragan-Kelley, B., Pérez, F., et al. 2016, in in Positioning and Power in Academic Publishing: Players, Agents and Agendas, ed. F. Loizides \& B. Scmidt (Amsterdam: IOS Press), 87

Kumar, R., Carroll, C., Hartikainen, A., \& Martin, O. 2019, JOSS, 4, 1143

Lee, E. J., \& Chiang, E. 2016, ApJ, 817, 90

Lee, E. J., Chiang, E., \& Ormel, C. W. 2014, ApJ, 797, 95

Lendl, M., Triaud, A. H. M. J., Anderson, D. R., et al. 2014, A\&A, 568, A81

Li, G., \& Winn, J. N. 2016, ApJ, 818, 5

Lightkurve Collaboration, Cardoso, J. V. d. M., Hedges, C., et al. 2018, Lightkurve: Kepler and TESS time series analysis in Python, Astrophysics Source Code Library, ascl: 1812.013

Lin, D. N. C., Bodenheimer, P., \& Richardson, D. C. 1996, Natur, 380, 606 Lin, D. N. C., \& Papaloizou, J. 1986, ApJ, 309, 846 
Luger, R., Agol, E., Foreman-Mackey, D., et al. 2019, AJ, 157, 64

Mandel, K., \& Agol, E. 2002, ApJL, 580, L171

McKinney, W. 2010, Proc. 9th Python in Science Conf., ed. S. van der Walt \& J. Millman,, 56

Mireles, I., Shporer, A., Grieves, N., et al. 2020, AJ, 160, 133

Montalto, M., Borsato, L., Granata, V., et al. 2020, MNRAS, 498, 1726

Moorhead, A. V., Ford, E. B., Morehead, R. C., et al. 2011, ApJS, 197, 1

Naoz, S., Farr, W. M., \& Rasio, F. A. 2012, ApJL, 754, L36

Neal, R. M. 2012, arXiv:1206.1901

Newton, E. R., Mann, A. W., Tofflemire, B. M., et al. 2019, ApJL, 880 L17

Nielsen, L. D., Bouchy, F., Turner, O., et al. 2019, A\&A, 623, A100

Petrovich, C. 2015, ApJ, 805, 75

Petrovich, C., \& Tremaine, S. 2016, ApJ, 829, 132

Petrovich, C., Tremaine, S., \& Rafikov, R. 2014, ApJ, 786, 101

Queloz, D., Anderson, D. R., Collier Cameron, A., et al. 2010, A\&A, 517, L1

Rasio, F. A., \& Ford, E. B. 1996, Sci, 274, 954

Ricker, G. R., Winn, J. N., Vanderspek, R., et al. 2015, JATIS, 1, 014003

Rodriguez, J. E., Quinn, S. N., Huang, C. X., et al. 2019, AJ, 157, 191

Rodriguez, J. E., Quinn, S. N., Zhou, G., et al. 2021, AJ, 161, 194

Salvatier, J., Wiecki, T. V., \& Fonnesbeck, C. 2016, PeerJ Comput. Sci., 2, e55

Santerne, A., Moutou, C., Tsantaki, M., et al. 2016, A\&A, 587, A64

Schlecker, M., Kossakowski, D., Brahm, R., et al. 2020, AJ, 160, 275
Shabram, M., Demory, B.-O., Cisewski, J., Ford, E. B., \& Rogers, L. 2016, ApJ, 820, 93

Shallue, C. J., \& Vanderburg, A. 2018, AJ, 155, 94

Smith, A. M. S., Anderson, D. R., Armstrong, D. J., et al. 2014, A\&A, 570, A64

Socrates, A., Katz, B., Dong, S., \& Tremaine, S. 2012, ApJ, 750, 106

Stassun, K. G., Oelkers, R. J., Paegert, M., et al. 2019, AJ, 158, 138

Stassun, K. G., Oelkers, R. J., Pepper, J., et al. 2018, AJ, 156, 102

Stassun, K. G., \& Torres, G. 2018, ApJ, 862, 61

Teske, J. K., Thorngren, D., Fortney, J. J., Hinkel, N., \& Brewer, J. M. 2019 AJ, 158, 239

Thorngren, D. P., Fortney, J. J., Murray-Clay, R. A., \& Lopez, E. D. 2016, ApJ, 831, 64

Theano Development Team 2016, arXiv:abs/1605.02688

Tsang, D., Turner, N. J., \& Cumming, A. 2014, ApJ, 782, 113

Udry, S., Mayor, M., \& Santos, N. C. 2003, A\&A, 407, 369

van der Walt, S., Colbert, S. C., \& Varoquaux, G. 2011, CSE, 13, 22

Van Eylen, V., Albrecht, S., Huang, X., et al. 2019, AJ, 157, 61

Virtanen, P., Gommers, R., Oliphant, T. E., et al. 2020, NatMe, 17, 261

Winn, J. N. 2010, arXiv:1001.2010

Wittenmyer, R. A., O’Toole, S. J., Jones, H. R. A., et al. 2010, ApJ, 722, 1854

Wu, Y., \& Lithwick, Y. 2011, ApJ, 735, 109

Yu, L., Vanderburg, A., Huang, C., et al. 2019, AJ, 158, 25 